\title{
Importancia de los términos de intercambio en la economía colombiana
}

\author{
Andrés Felipe Oviedo Gómez y Lya Paola Sierra Suárez
}

\section{Resumen}

Dado que la mayoría de las exportaciones de Colombia consisten en materias primas, cuyos precios determinan directamente los términos de intercambio, se hace necesario un análisis detallado de los impactos de esa variable en la economía. En este trabajo se analizan los efectos de los choques en los términos de intercambio sobre la producción, la inversión, el consumo, la balanza comercial, el tipo de cambio real y la inflación en Colombia. Se emplea una extensa base de datos, compuesta por 129 variables relacionadas con la actividad económica correspondientes al período 2001-2016, y se estima un modelo FAVAR. Los resultados sugieren que los choques en los términos de intercambio generan efectos significativos sobre la economía colombiana, ya que explican aproximadamente un $5 \%$ de la variación de la actividad económica del país, un $8 \%$ de la inversión, un 6\% de la balanza comercial y un $1 \%$ del movimiento en el tipo de cambio real.

\section{Palabras clave}

Relación de intercambio, comercio internacional, condiciones económicas, desarrollo económico, modelos econométricos, Colombia

\section{Clasificación JEL}

F14, F41, O54

\section{Autores}

Andrés Felipe Oviedo Gómez es profesor de la Universidad del Valle, Colombia. Correo electrónico: oviedo.andres@correounivalle.edu.co.

Lya Paola Sierra Suárez es Profesora Asociada en el Departamento de Economía de la Pontificia Universidad Javeriana de Cali (Colombia). Correo electrónico: lyap@javerianacali.edu.co. 


\section{Introducción}

Los términos de intercambio representan la razón entre el precio de las exportaciones y el precio de las importaciones de un país, relación que expresa la capacidad de compra que tienen los productos nacionales vendidos en el extranjero. Al ser una variable exógena, los términos de intercambio permiten observar los efectos de choques externos sobre los diferentes sectores económicos.

A diferencia de los países industrializados, un alto porcentaje de las exportaciones de muchos países en desarrollo consiste en materias primas o productos básicos, lo que los hace más vulnerables a choques en los términos de intercambio. De acuerdo a Fernández, González y Rodríguez (2015), la proporción promedio de materias primas exportadas por países en desarrollo es superior al doble de las exportadas por los países industrializados. Por su propia naturaleza, los precios de los productos básicos son altamente volátiles, debido a que presentan demandas y ofertas inelásticas ${ }^{1}$ en el corto plazo (Sinnott, Nash y De la Torre, 2010). Según los autores mencionados, las mayores fluctuaciones de los términos de intercambio ocurren en países que concentran sus exportaciones en bienes minero-energéticos, seguidos por exportadores de otros bienes primarios y por exportadores de manufacturas. Los efectos económicos que los choques en los términos de intercambio generan en las economías no se derivan exclusivamente de su magnitud y del tipo de producto exportado, sino también del grado de apertura de la economía a los mercados internacionales. Para Ortiz (2016), desde la década de 1990 varios países de América del Sur han implementado el modelo neoliberal sin un proceso de diversificación productiva, lo cual ha elevado el grado de exposición de esas economías a las perturbaciones externas. A raíz de ello, los efectos de los choques se han hecho sentir de forma inmediata en el ingreso público y en los agregados fiscales (Banco Mundial, 2000).

Para Colombia los choques en los términos de intercambio son de suma importancia, dado que en 2015 las exportaciones representaron el 15\% del PIB (Banco Mundial, 2016) y estuvieron constituidas en un $80 \%$ por productos básicos: el petróleo (40\%), el carbón (13\%), el café (7\%) y el níquel (1\%) representaron más de la mitad de las exportaciones nacionales².

El objetivo de este artículo es cuantificar y entender los efectos ocasionados por los choques en los términos de intercambio sobre las variables macroeconómicas, principalmente la producción, la inversión, el consumo, la balanza comercial, el tipo de cambio real y la inflación en Colombia. Se utiliza una metodología que combina los modelos de vectores autorregresivos con los modelos factoriales dinámicos. Esta metodología fue propuesta por Bernanke, Boivin y Eliasz (2005) y recibe el nombre de vector autorregresivo con factor aumentado (factor augmented VAR (FAVAR)). Los modelos FAVAR tienen la ventaja de utilizar un sinnúmero de variables macroeconómicas y eliminan las limitaciones de información de los modelos VAR, lo que garantiza un análisis más amplio de los efectos que tienen los términos de intercambio sobre toda la actividad económica; además, reducen las anomalías que pueden generar los sesgos de variables omitidas.

Para este análisis, además de las variables de interés se emplearon 129 series macroeconómicas trimestrales correspondientes al período 2001-2016. Dado que hasta el momento no se conocen estudios empíricos que hagan uso de los modelos FAVAR, ni de una utilización tan extensa de las variables económicas en el análisis de los choques en los términos de intercambio, la principal contribución de este artículo consiste en brindar una perspectiva más amplia acerca de la importancia relativa de los términos de intercambio sobre las diferentes variables macroeconómicas en Colombia.

El trabajo está dividido en las siguientes secciones, que siguen a la introducción: en la sección II se hace una reseña de los estudios previos tanto a nivel internacional como de Colombia; en la

\footnotetext{
1 Véase más información sobre elasticidad de la oferta y la demanda en Roberts y Schlenker (2013).

2 La información se obtuvo del Departamento Administrativo Nacional de Estadística (DANE).
} 
sección III se definen los términos de intercambio, se explica el método de construcción y se observa la evolución de dichos términos durante los últimos años, así como su relación con las principales variables macroeconómicas en Colombia; la sección IV contiene una descripción de la metodología empírica aplicada; en la sección $V$ se presenta la descripción de los datos y en la sección VI los resultados empíricos. Por último, en la sección VII se exponen las conclusiones finales.

\section{Revisión de los estudios realizados}

En esta sección se hace una reseña de los estudios que han llevado a cabo diferentes autores relativos a la influencia de los choques en los términos de intercambio de las economías desarrolladas y emergentes cuyas exportaciones están compuestas en un porcentaje importante por materias primas.

Partiendo de la premisa de que los países exportadores de productos básicos son más propensos a sentir los efectos de los choques en los términos de intercambio, varios autores han observado que diferentes agregados económicos como el PIB, la inversión, el consumo y el tipo de cambio son las variables que se ven mayormente afectadas, pero que la magnitud del impacto está condicionada por las características económicas del país o por la metodología aplicada. En la década de 1990, Mendoza (1995) examinó la relación entre los términos de intercambio y los ciclos de negocios, utilizando un modelo teórico aplicado a 23 países en desarrollo y a 7 países industrializados. Los resultados muestran que para los países emergentes, los choques en los términos de intercambio explican entre el $37 \%$ y el $56 \%$ de la variabilidad del PIB real, mientras que en los países industrializados ascienden al 33\%. Por su parte, Schmitt-Grohé y Uribe (2015) elaboraron un modelo similar al de Mendoza (1995) y estimaron un vector autorregresivo estructural (SVAR) para evaluar el efecto de los términos de intercambio respecto a 38 países pobres y en desarrollo. El modelo teórico indica que un choque resultante de dichos términos explica en promedio un 30\% de la variación de la producción, el consumo y la inversión de esos países. Por el contrario, los resultados empíricos del SVAR muestran una variación de aproximadamente el 10\% sobre dichos agregados. Estos resultados son coherentes con los resultados a que llegó Broda (2004), quien concluyó, a partir del análisis de 75 países en desarrollo, que los términos de intercambio generan una volatilidad de menos del $10 \%$ del PIB real.

La evidencia muestra un mayor impacto de los choques de los términos de intercambio en países en desarrollo que concentran sus exportaciones en productos básicos, aunque no es despreciable el efecto de las variaciones de los precios de esos productos en los países desarrollados exportadores de petróleo. Andrews y Rees (2009) hicieron un estudio para observar los efectos de los términos de intercambio sobre la economía australiana y llegaron a la conclusión de que las variaciones positivas y superiores a una desviación estándar de dichos términos generaban una volatilidad anual del 1,1\% del crecimiento del PIB y del 1,2\% de la inflación, respectivamente. Por su parte, y también con respecto a Australia, Jääskelä y Smith (2013) emplearon un modelo VAR para observar la respuesta de la inflación, la producción, la tasa de interés y la tasa de cambio ante tres tipos de choques exógenos transmitidos por el canal de los términos de intercambio. Los resultados muestran que los términos de intercambio explican las dos terceras partes de la variación de la tasa de cambio, pero su influencia sobre las demás variables es inferior a la quinta parte. Con base en la metodología anterior, Karagedikli y Price (2013) consideraron que los choques exógenos transmitidos a través de los términos de intercambio explican alrededor del $20 \%$ de las fluctuaciones del PIB en Nueva Zelandia. Por su parte, para el caso del Canadá, Charnavoki y Dolado (2014) propusieron un modelo factorial dinámico estructural para identificar los principales choques externos que impulsan los precios mundiales de las materias primas. Los resultados indican que ante un choque positivo de la demanda mundial, los términos de intercambio presentan una mejora del 0,04\%. 
América Latina es especialmente propensa a sufrir los efectos de choques en los términos de intercambio ya que la mayoría de los países de la región son dependientes de las materias primas. En 2014 la participación de los productos básicos en las exportaciones totales de la región superó el 80\%. Según Ben Zeev, Pappa y Vicondoa (2016) con base en el modelo teórico de Schmitt-Grohé y Uribe (2015) y un modelo VAR, los términos de intercambio explican en promedio el $25 \%$ de las fluctuaciones de la producción, tanto a nivel teórico como empírico, de la Argentina, el Brasil, Chile, Colombia, el Ecuador, México y el Perú. Por su parte, Izquierdo, Romero y Talvi (2008) estudiaron las fluctuaciones de los términos de intercambio para la Argentina, el Brasil, Chile, Colombia, México, el Perú y Venezuela (República Bolivariana de) y llegaron a la conclusión de que un incremento de dos puntos porcentuales en los términos de intercambio genera un aumento del crecimiento del PIB de solo el 0,21\% durante el segundo trimestre después del choque. En cambio, Ahmed (2003) encontró que las variaciones en los términos de intercambio explican en promedio menos del 8\% de las fluctuaciones de la producción de la Argentina, el Brasil, Chile, Colombia, México y Venezuela (República Bolivariana de), excluyendo al Ecuador. Por otro lado, por medio de un proceso de descomposición de varianza para el Perú, Castillo y Salas (2012) llegaron a la conclusión de que los choques en los términos de intercambio explican aproximadamente el $90 \%$ de las fluctuaciones del producto, la inversión y el consumo en un horizonte de 10 años.

A pesar de la importancia de los términos de intercambio en economías dependientes de materias primas, en Colombia existen pocos estudios que evalúen la incidencia de los choques en los términos de intercambio sobre la actividad económica. En general, en la literatura especializada se evalúan los efectos de choques en los términos de intercambio sobre variables macroeconómicas por separado. Por ejemplo, Arteaga, Granados y Joya (2013) analizan los efectos de los términos de intercambio en la tasa de cambio real; por su parte, Gaviria (1993) estudia la incidencia de los choques en los términos de intercambio sobre el ahorro privado; y Parra (2008) y Hernández (2013) evalúan las fluctuaciones de los términos de intercambio sobre el PIB de la economía colombiana. Es importante resaltar el trabajo llevado a cabo por este último autor, quien llegó a la conclusión de que entre un $27 \%$ y un $33 \%$ de la variabilidad trimestral del PIB depende de los términos de intercambio. Como se puede apreciar, aunque existen trabajos que analizan los impactos de los términos de intercambio sobre diferentes variables, por ahora no se dispone de ningún estudio que utilice un extenso conjunto de variables que describan en gran medida la actividad económica nacional y que evalué sus respuestas ante las variaciones de dichos términos. Es este el vacío que se pretende cubrir con este trabajo de investigación, a partir de la estimación de un modelo FAVAR que, a diferencia de los modelos de vectores autorregresivos, permita la estimación de la respuesta dinámica de un gran número de variables internas ante una innovación externa (Mumtaz y Surico, 2009). Además, se busca brindar otra alternativa a la desconexión entre los modelos teóricos y los modelos empíricos: así lo han planteado Schmitt-Grohé y Uribe (2015) y Aguirre (2011), quienes aconsejan la aplicación de otra metodología que garantice una mejor interpretación de los datos.

\section{Los términos de intercambio y la economía colombiana}

A continuación se presenta un análisis descriptivo de la evolución de los términos de intercambio en Colombia y de las variables que reciben el mayor impacto de los choques de dichos términos, realizado por autores como Hernández (2013), Schmitt-Grohé y Uribe (2015) y Castillo y Salas (2012).

Los datos que se usarán para modelar los términos de intercambio provienen del índice elaborado por el banco central de Colombia (Banco de la República), a través de precios implícitos y a partir de la metodología del índice de Paasche encadenado propuesta por Garavito y otros (2011). La 
ecuación (1) muestra la estructura general del índice de términos de intercambio (ITI) por medio de la razón entre los índices encadenados de Paasche de precios de exportaciones $\left(I P_{X, t}\right)$ e importaciones $\left(I P_{M, t}\right)$, para un período $t$ :

$$
I T I_{t}=\left(\frac{I P_{X, t}}{I P_{M, t}}\right) \cdot 100
$$

Con base en la metodología citada, el gráfico 1 muestra el índice de términos de intercambio de Colombia. El hecho más significativo que se puede observar en el gráfico es la mejora de los términos de intercambio a partir de la década de 2000. Para autores como Jenkins (2011) y Sierra (2015), ello se debió al auge de los precios de las materias primas resultante del incremento de la demanda por parte de China y la India y a la caída de los precios mundiales de las manufacturas en la primera década del siglo XXI. Este auge en los términos de intercambio en la mayoría de los países latinoamericanos los convirtió en los proveedores más importantes de los países asiáticos. También se aprecia un deterioro de los términos de intercambio a mediados de 2008 a raíz de la crisis financiera global, y otra posterior disminución en 2014 ocasionada por la pronunciada caída del precio del petróleo.

Gráfico 1

Colombia: índices de los términos de intercambio, 1995-2016

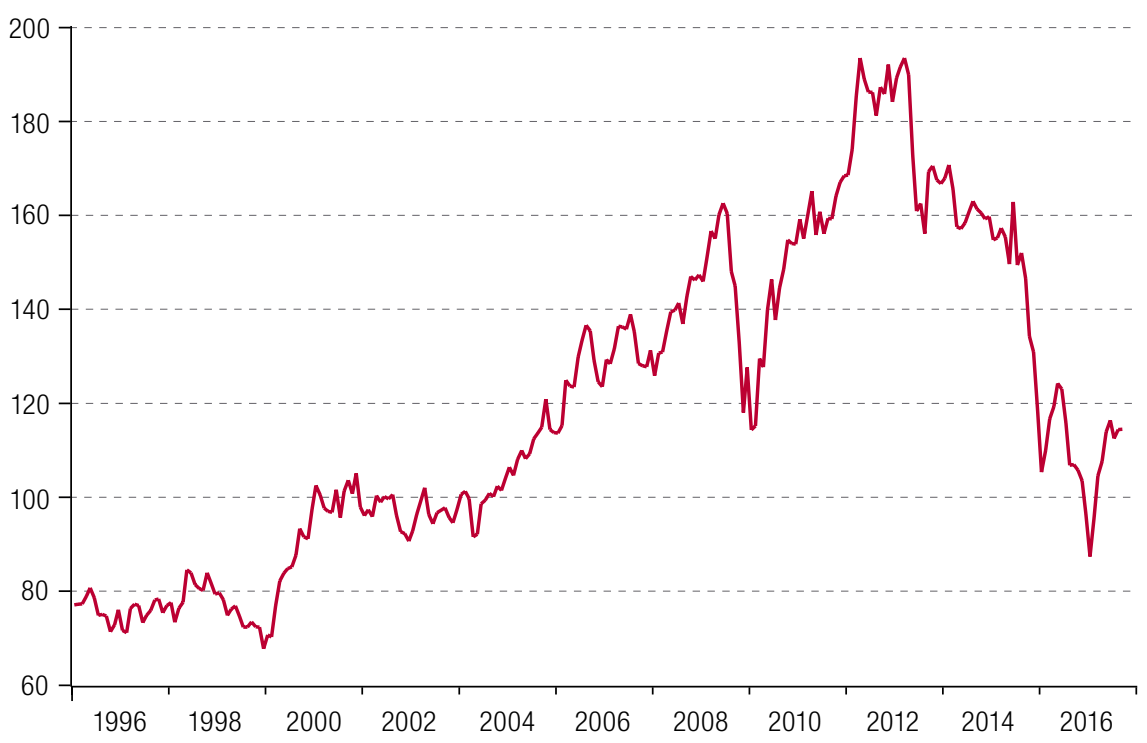

Fuente: Banco de la República de Colombia, 2016.

Dada la importancia de las materias primas en la composición de las exportaciones de Colombia, se analiza gráficamente la relación de sus precios con los términos de intercambio. En el gráfico 2 se indican los precios del café (línea discontinua) y los precios del petróleo ${ }^{4}$ (línea continua) en Colombia durante el período 1980-2016. Se señalan esos productos porque en las últimas décadas han sido las materias primas con mayor peso en las exportaciones colombianas. Gracias a la bonanza cafetera, en la década de 1970 el café se convirtió en el producto insignia para la economía nacional, ya que representaba más del $50 \%$ de las exportaciones totales (60\% a finales de los años setenta) (Cano y

3 Los precios del café se toman de cafés arábicos suaves de Colombia, precios en efectivo de la Organización Internacional de Café, Nueva York, en dólares por libra.

4 Los precios del petróleo se toman del promedio simple de tres precios al contado: Dated Brent, WTI y el Dubái Fateth, en dólares por barril. 
otros, 2012). Entre 1980 y 2016 se observa una correlación de 0,57 entre el índice de términos de comercio y los precios del café, mientras que la correlación con los precios del petróleo fue de 0,37. Sin embargo, a medidos de la década de 1990 el petróleo aumentó significativamente su participación en las exportaciones y desde 1995 ha sido el principal producto exportado por Colombia. Cabe observar que en las últimas tres décadas (1995-2016) calculamos una correlación entre los términos de intercambio y los precios del petróleo positiva que asciende a 0,88 , mientras que la correlación con los precios del café es del 0,65. Ello permite inferir que las oscilaciones del precio del petróleo han ganado relevancia en la evaluación de los términos de intercambio en Colombia.

\section{Gráfico 2}

Comportamiento de los precios del petróleo y el café, 1980-2016

(En dólares por barril y dólares por libra)

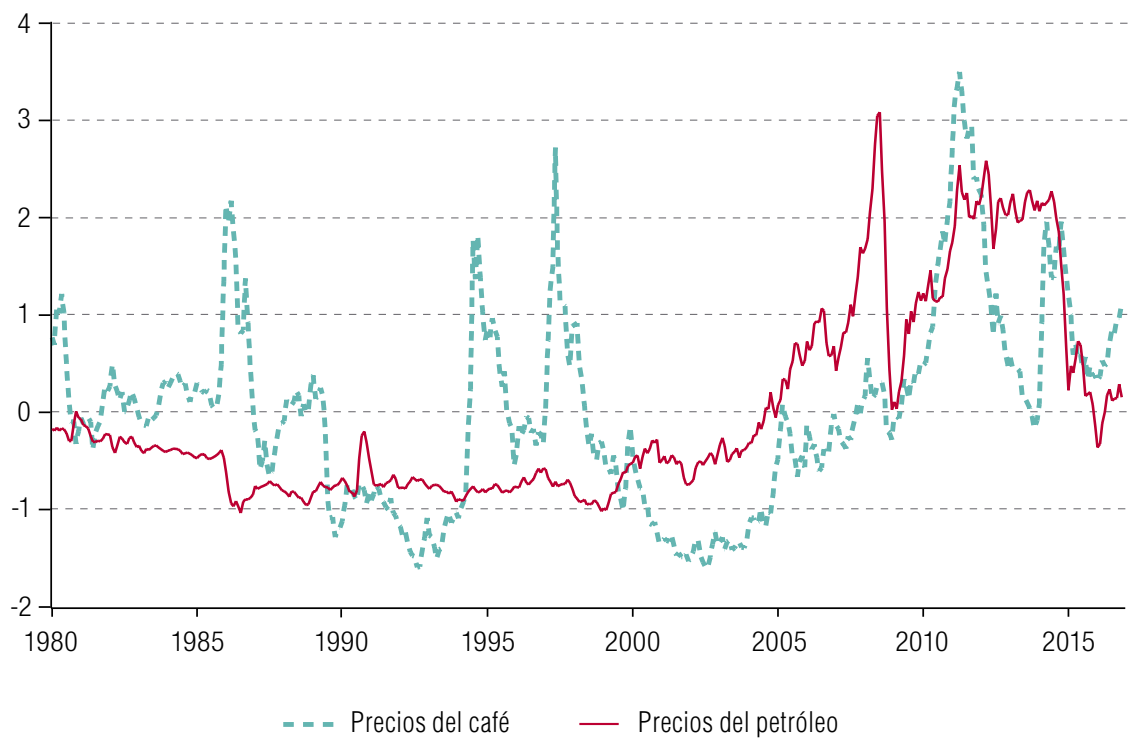

Fuente: Fondo Monetario Internacional (FMI).

En relación con las otras materias primas exportadas por Colombia, como el carbón y el níquel, calculamos una correlación de los precios del níquel y el carbón con los términos de intercambio de 0,4 y 0,7, respectivamente, para el período 1980-2016.

De acuerdo con la literatura anteriormente citada, también se ven afectadas por la evolución de los términos de intercambio las siguientes variables: el PIB, la inversión (formación bruta de capital fijo), el consumo total, la balanza comercial, el índice de tasa de cambio real y la inflación total. El gráfico 3 muestra la evolución de estas variables en los últimos años. Para el período 2001-2016 calculamos una correlación entre los términos de intercambio y las variables de interés y se percibió que el PIB presentaba una correlación positiva de 0,5, valor cercano al 0,35 hallado por Hernández (2013). Además, se fijó una correlación positiva entre los términos de intercambio y la inversión y el consumo del 0,59 y 0,54, respectivamente. Parra (2008) afirma que los choques en los términos de intercambio generan incrementos en la inversión y en el consumo debido a la reasignación del capital a su lugar productivo. Por su parte, en el caso de la balanza comercial se calculó una correlación elevada y positiva del 0,82 y para el índice de tasa de cambio real se determinó una relación negativa de 0,9. Este resultado concuerda con el obtenido por Poncela, Senra y Sierra (2017), quienes infieren, por ejemplo, que el ingreso derivado del aumento del precio del petróleo genera una entrada de divisas que da lugar a una apreciación real del peso frente al dólar. Finalmente, nuestro cálculo de la correlación entre los términos de intercambio y la inflación refleja una correlación negativa de 0,7. 


\section{Gráfico 3}

Evolución de las series de interés, 2001-2016

A. PIB

(En miles de millones de pesos a precios constantes de 2005)

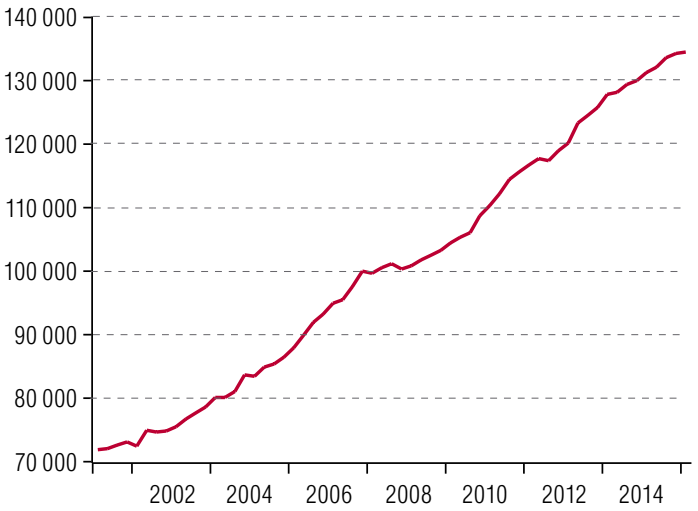

C. Balanza comercial

(En billones de pesos)

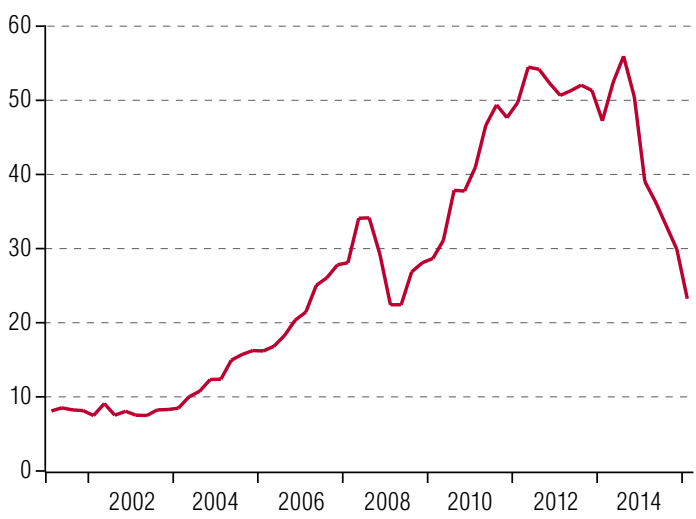

E. Inflación

(Variación anual del IPC ponderado)

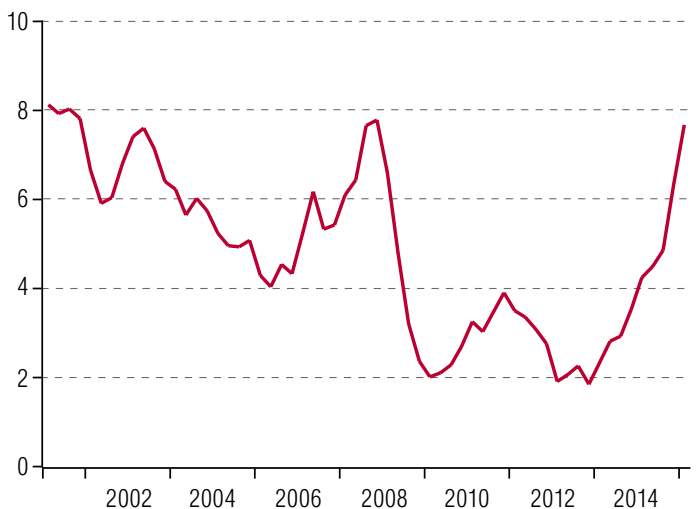

B. Inversión en formación bruta del capital fijo (En miles de millones de pesos)

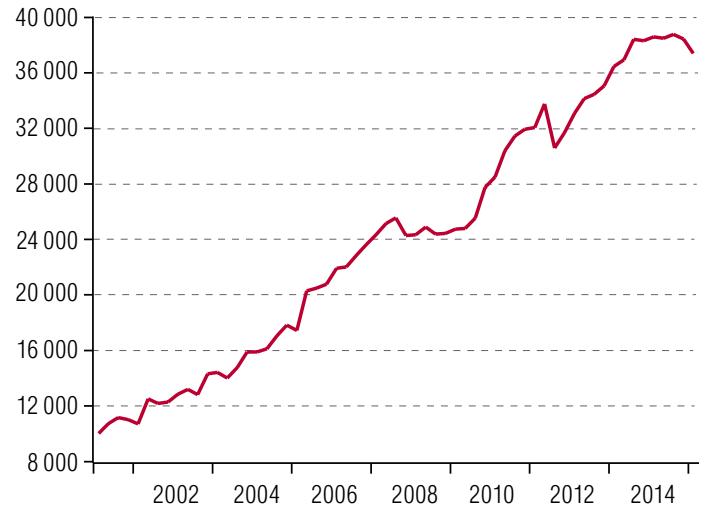

D. Índice de tasa de cambio real $(2010=100))$

(Respecto a 18 países miembros del Fondo Monetario Internacional (FMI))

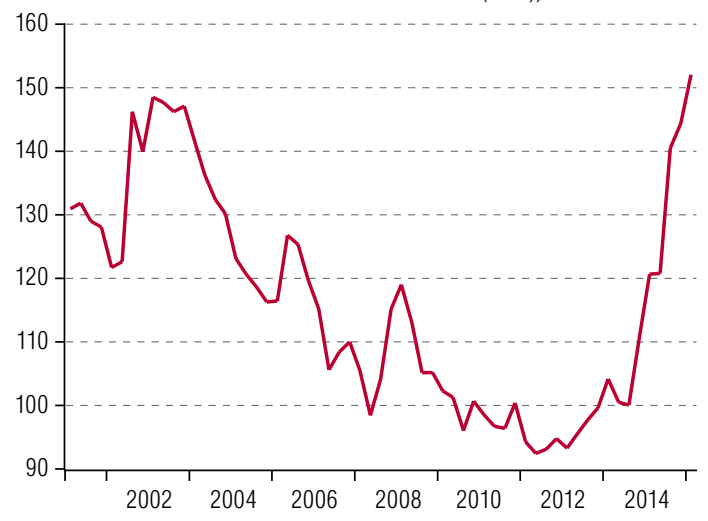

F. Consumo total

(En miles de millones de pesos)

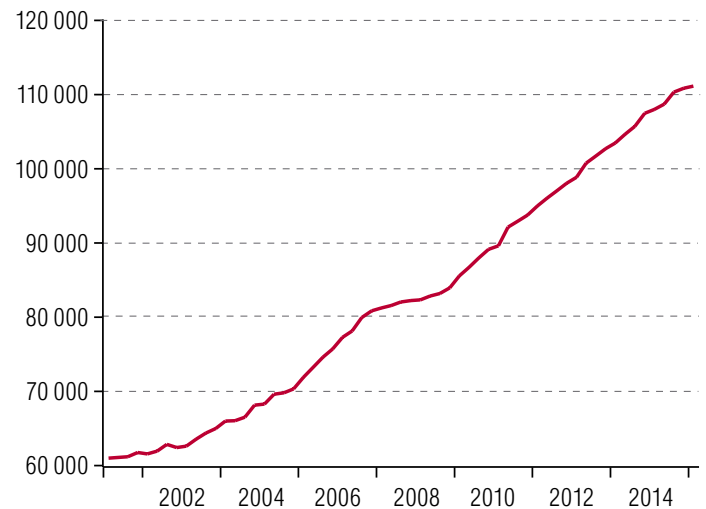

Fuente: Banco de la República de Colombia y Departamento Administrativo Nacional de Estadística (DANE). 
A continuación se explica el mecanismo de transmisión de cómo un choque positivo en los términos de intercambio afecta a cada una de las variables.

Dada la relación que presentan los términos de intercambio con los diferentes agregados económicos colombianos, y como consecuencia de su exogeneidad, un choque positivo en los términos de intercambio debido, por ejemplo, al incremento de los precios de los productos exportados (productos básicos) genera una entrada de divisas extranjeras al país. Ello a su vez incentiva la solicitud de créditos, sobre todo en el sector beneficiado por el auge, como el de las materias primas, y por consiguiente favorece la inversión. El mayor ingreso de divisas al país favorece a todos los sectores productores de bienes básicos (directos e indirectos) y al consumo, y como indica Hernández (2013), incrementa las utilidades del sector financiero y del gobierno (mayores ingresos fiscales). La moneda local se aprecia en términos reales, en primer lugar debido a la apreciación nominal, ocasionada por la entrada de divisas, y en segundo lugar por el incremento de los precios, resultante del aumento de la demanda en general. Un efecto indirecto negativo, muchas veces dejado de lado en el análisis de los impactos de los términos de intercambio, es el efecto que puede causar la apreciación real de la moneda en la competitividad del sector exportador, que produce una disminución del valor agregado y en el empleo en dichos sectores (véanse Sierra y Manrique, 2014; Peláez y Sierra, 2016). La metodología que se plantea para este estudio permite incorporar todas las interacciones de la economía con efectos positivos y negativos, dependiente del sector de que se trate, ante un choque positivo en los términos de intercambio, así como detectar el efecto neto del choque en la economía en general.

\section{Metodología}

En el siguiente apartado se presenta una descripción de la metodología empírica que se utilizó como herramienta para observar los efectos que tienen los choques en los términos de intercambio sobre las diferentes variables macroeconómicas nacionales. Se emplea la metodología FAVAR de Bernanke, Boivin y Eliasz (2005), ya que esta permite evaluar la persistencia temporal de los choques exógenos a través de las funciones impulso-respuesta de un gran número de variables macroeconómicas. A diferencia de los modelos VAR, incorpora factores que brindan información suficiente para capturar de forma general y precisar los movimientos simultáneos de la actividad económica. Además, reducen parcialmente las dificultades de dimensionalidad.

\section{Modelos FAVAR}

Se define un vector $Y_{t}$ de dimensiones $M x 1$, que está compuesto por variables económicas observables o de interés: términos de intercambio, producto, inversión, consumo, balanza comercial, tipo de cambio e inflación, comprendidas para un $t=1,2, \ldots T$ períodos. Se infiere, como normalmente sucede en los modelos VAR, que existe información adicional que no figura en $Y_{t}$ y que es relevante para explicar la dinámica de las series de este vector. Sin embargo, dicho conjunto de datos excluidos por las limitantes del modelo empírico puede resumirse en un vector $K x 1$ de factores no observables, $F_{t}$ La dinámica de $\left(F_{t^{\prime}} Y_{t}\right)$ puede ser representada por un modelo VAR, tal como se expresa a continuación en la ecuación (2):

$$
\left[\begin{array}{l}
F_{t} \\
Y_{t}
\end{array}\right]=\Phi(L)\left[\begin{array}{c}
F_{t-1} \\
Y_{t-1}
\end{array}\right]+v_{t}
$$

donde $\Phi(L)$ es una matriz que representa el polinomio del operador de rezagos finito de orden $d$ y el termino de error, $v_{t}$, tiene media cero y una matriz de covarianzas $Q$. 
Para la estimación de los factores, $F_{t}$, se utilizan los llamados modelos factoriales dinámicos, propuestos originalmente por Geweke (1976) y desarrollados ampliamente por Stock y Watson (2002, 2005, 2011). Dicho modelo se puede representar de forma estática como:

$$
X_{t}=\Lambda F_{t}+e_{t}
$$

siendo $X_{t}$ un vector de dimensión $N x 1$ que contiene un amplio conjunto de datos (129 series de tiempo observables, donde no se incluyen las variables de interés). Se asume que este vector de información $X_{t}$ puede ser representado como la suma de dos componentes no observables: uno o unos componentes comunes a todas las variables, $F_{t}$, y un componente idiosincrático, $e_{t}$, propio de cada variable. En la ecuación (3) $\Lambda$ es una matriz compuesta por las cargas de los factores, de dimensión $N x K$ (el término $\Lambda F_{t}$ se denomina componentes comunes de $X_{t}$ ) y el término de error, $e_{t}$ tiene media cero y distribución normal, con dimensión $N x 1$. Este último término puede presentar una escasa correlación transversal, en la medida que captura el componente idiosincrático ${ }^{5}$.

Siguiendo a Bernanke, Boivin y Eliasz (2005), la estimación del modelo FAVAR se realizará en dos etapas: la primera consiste en la estimación de los espacios que ocupan los factores, por medio de $k$ componentes principales de $X_{t}$, y la segunda etapa estima la ecuación (2) a través del método estándar de vectores autorregresivos, lo que requiere reemplazar $F_{t}$ por $\hat{F}_{t}$ (factores estimados). Este método se caracteriza por su simplicidad computacional y por su alto grado de robustez para un nivel de correlación transversal en el error (Stock y Watson, 2005). Además, Forni y otros (2000, 2005) demostraron la coherencia del estimador por componentes principales cuando el número de series $N$ y la dimensión $T$ tienden al infinito. Por su parte, Bai y $\mathrm{Ng}$ (2002) afirman que las estimaciones son coherentes cuando el min $\{N, T\}$ es igual o mayor a 40.

Para determinar el número de factores que se incluirán en la segunda etapa de la estimación se utilizarán los criterios de información propuestos por Bai y Ng (2002). Además, se estimarán las funciones impulso-respuesta generalizadas que propusieron Pesaran y Shin (1998): a diferencia de las funciones impulso-respuesta halladas por el método de Cholesky, estas no dependen de la ordenación del VAR.

\section{Datos}

La base de datos utilizada está compuesta por las series de interés descritas (términos de intercambio, producto, inversión, consumo, balanza comercial, tipo de cambio e inflación) y 129 series macroeconómicas que representan diferentes categorías de la economía colombiana. Entre paréntesis se indica la cantidad de variables por categoría: actividad económica (23), sector externo (23), tipo de cambio real (5), precios (31), agregados monetarios (16), empleo total nacional (6), sector público no financiero (7), flujos de inversión extranjera en Colombia (12) y tasas de interés (6). El estudio abarcó un panel equilibrado, compuesto por datos trimestrales comprendidos en el período 2001-2016. La elección del período estuvo determinada primero por la disponibilidad de los datos sin cambios metodológicos y porque excluye el punto de ruptura presentado en los años noventa por la apertura económica colombiana, que aumentó la exposición a los choques exógenos. El período posterior a 2001 es también interesante porque abarca el efecto que tiene en los términos de intercambio de los países latinoamericanos el ingreso de China a la Organización Mundial de Comercio (OMC) en 2001, como se indica en la sección III. Ese período se caracteriza por un alto crecimiento de las economías asiáticas, principalmente de China, que impulsaron los términos de intercambio de los países latinoamericanos (Sierra, 2015).

5 Tanto $F_{t}$ como $e_{t}$ pueden seguir un proceso auto-regresivo, aunque en esta investigación no se tiene en cuenta. 
Antes de efectuar las transformaciones de las series se eliminaron los datos atípicos (outliers), siguiendo a Stock y Watson (2002), quienes afirman que esos datos alteran el método de estimación de los factores estimados por componentes principales, ya que aumentan significativamente el valor de la varianza. Por lo tanto se utiliza el programa de regresión de series de tiempo con ruido ARIMA, observaciones perdidas y atípicos o TRAMO (time series regression with ARIMA noise, missing values and outliers), desarrollado por Gómez y Maravall (1998), para hallar de forma automática los valores atípicos. Este método utiliza la especificación: $\ln X_{i, t}=\frac{\theta_{i}(B)}{\Delta \phi_{i}(B)} \varepsilon_{i t}+$ outliers, donde $\Phi_{i}(B)$ es un polinomio autorregresivo de orden $p_{i^{\prime}} \theta_{i}(B)$ es un polinomio de medio móvil de orden $q_{i}$ con el operador de rezagos $B$ y $\Delta$ es el operador de diferencias (1-B).

El modelo descrito en la sección IV explica que la matriz de variables $X_{t}$ debe ser estacionaria, por lo que antes de la estimación del modelo se aplicó el test de raíces unitarias con intercepto y tendencia, utilizando las pruebas sugeridas por Dickey y Fuller (1979) y Phillips y Perron (1988). En el anexo A1 figura la información de las variables de interés y las 129 series utilizadas con su respectiva transformación. Por último, las series, tras ser convertidas a estacionarias, son estandarizadas para que tengan media igual a cero y varianza igual a uno, siguiendo a Stock y Watson (2011).

\section{Resultados empíricos}

En esta sección se exponen los resultados obtenidos tras la estimación del modelo FAVAR (ecuación (2)) descrito en la sección IV. En dicha estimación se definen las variables observables $Y_{t}$ y se extraen los factores no observables $F_{t}$, de las 129 series restantes. La sección se divide en cuatro subsecciones: la estimación de los factores, las funciones impulso-respuesta, la descomposición de varianza y las pruebas de robustez.

\section{Estimación del número de factores}

Con base en las tres funciones de penalización propuestas por Bai y $\mathrm{Ng}$ (2002), se observa que las dos primeras indican la inclusión de cuatro factores, mientras que la tercera explica la utilización de nueve factores. En el cuadro 1 se resumen los resultados de los criterios de información.

Cuadro 1

Número de factores estimados utilizando los criterios de información de Bai y Ng (2002)

\begin{tabular}{cccccc}
\hline Muestra & Período & Número de observaciones & $I C_{1}$ & $I C_{2}$ & $I C_{3}$ \\
\hline Completa & 2001Trim 1-2016 Trim 1 & 61 & 4 & 4 & 9 \\
\hline
\end{tabular}

Fuente: Elaboración propia.

Nota: IC significa "criterios de información".

Como se ha indicado, del bloque de variables restantes (129) y por medio del análisis de los componente principales se escoge el primer componente principal, que explica aproximadamente el $18 \%$ de la proporción de varianza de este conjunto, tal como se señala en el cuadro 2 y se conoce como factor 1 o tipo de cambio real, debido al peso que tiene sobre las series y la correlación de 0,7 con el índice de tipo de cambio real. El siguiente factor explica aproximadamente un $16 \%$ de la proporción de varianza y se denomina factor 2 o agregados monetarios, debido al peso que ejerce sobre los agregados monetarios y la correlación positiva de 0,7 con la variable M1. Por su parte, el factor 3 , que explica aproximadamente un $13 \%$ de la varianza, corresponde a los precios ante la correlación positiva de 0,5 con las series de índice de precios al consumidor e índice de precios al productor. El factor 4, que explica aproximadamente un $10 \%$ de la varianza, se refiere a la actividad económica producto de la relación positiva de 0,5 con las variables de consumo y de crecimiento del PIB. Es evidente que los 
primeros cuatro factores descritos explican casi un $57 \%$ de la varianza total. A partir del quinto factor el aporte de cado uno es menor al 5\%, lo que corrobora la elección de cuatro factores.

Cuadro 2

Factores hallados por el análisis por componentes principales

\begin{tabular}{cccccc}
\hline No. del factor & Valor & Diferencia & Proporción & Valor acumulativo & $\begin{array}{c}\text { Proporción } \\
\text { acumulativa }\end{array}$ \\
\hline 1 & 18,117920 & 2,40323 & 0,1342 & 18,11792 & 0,1342 \\
\hline 2 & 15,714690 & 2,41727 & 0,1164 & 33,83262 & 0,2506 \\
\hline 3 & 13,297420 & 3,52985 & 0,0985 & 47,13004 & 0,3491 \\
\hline 4 & 9,767571 & 3,08406 & 0,0724 & 56,89761 & 0,4215 \\
\hline 5 & 6,683512 & 0,945658 & 0,0495 & 63,58112 & 0,4710 \\
\hline 6 & 5,737855 & 0,587268 & 0,0425 & 69,31898 & 0,5135 \\
\hline 7 & 5,150586 & 0,624079 & 0,0382 & 74,46956 & 0,5516 \\
\hline
\end{tabular}

Fuente: Elaboración propia.

\section{Funciones impulso-respuesta}

En los gráficos 4, 5 y 6 se observan los resultados de la estimación de las funciones impulso-respuesta generalizadas y acumuladas, generadas por un choque positivo en el índice de términos de intercambio $\left(\right.$ ITI $\left._{t}\right)$ sobre tres series macroeconómicas observables (inversión, $I_{t}$; balanza comercial, $B C_{t}$; y consumo total, $C_{t}$ ) con un intervalo de confianza del $95 \%$ y para un período de 10 trimestres. Para ello se emplea la especificación de cuatro factores (tipo de cambio real, agregados monetarios, precios y actividad económica) y tres rezagos, dado que para este valor el modelo es robusto y consistente en la evaluación de los supuestos sobre los residuales (véase el anexo A2). Los resultados ponen de manifiesto un efecto positivo y significativo de los términos de intercambio sobre la actividad económica (factor que simula el PIB), la inversión y la balanza comercial. Por el contrario, respecto al tipo de cambio real (factor que simula el índice de tipo de cambio real) se observa un efecto negativo y significativo, mientras que para los precios (factor que representa la inflación) y el consumo total no se aprecian efectos significativos.

\section{Gráfico 4}

Impulso-respuesta generalizada y acumulada del factor de actividad económica y la inversión ante un choque de una desviación estándar del índice de términos de intercambio

A. Respuesta acumulada de la actividad económica ante una innovación del índice de términos de intercambio

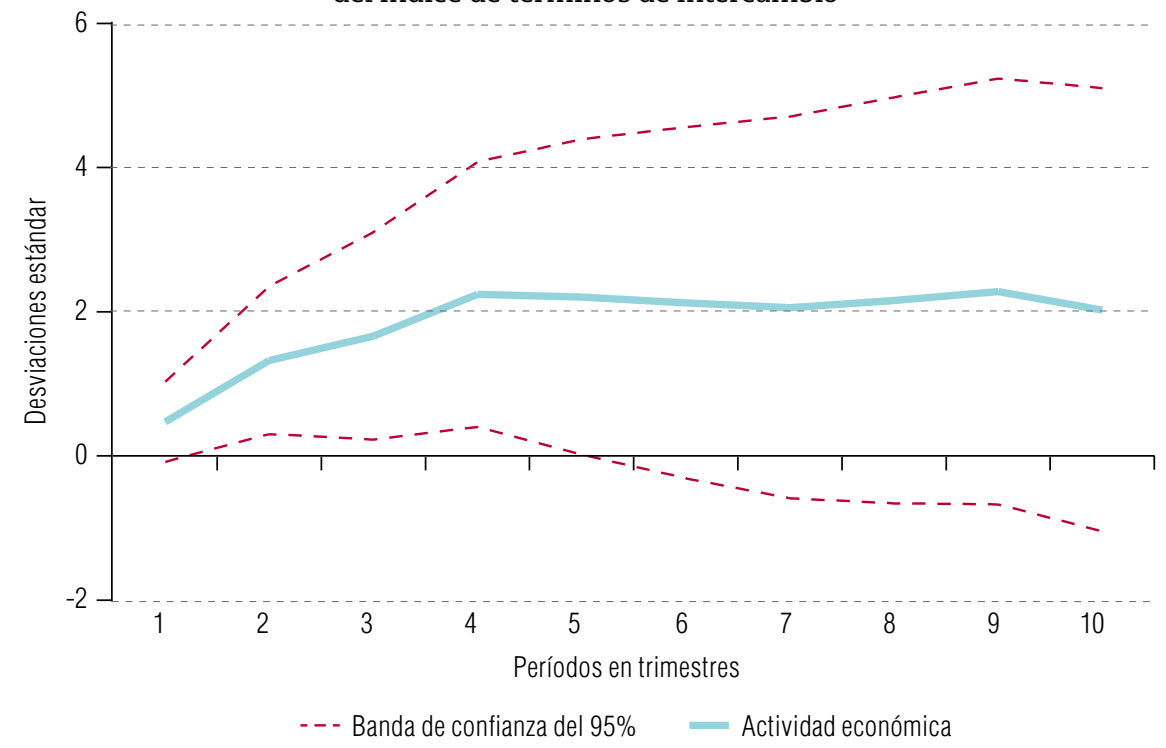


Gráfico 4 (conclusión)

B. Respuesta acumulada del tipo de cambio ante una innovación del índice de términos de intercambio

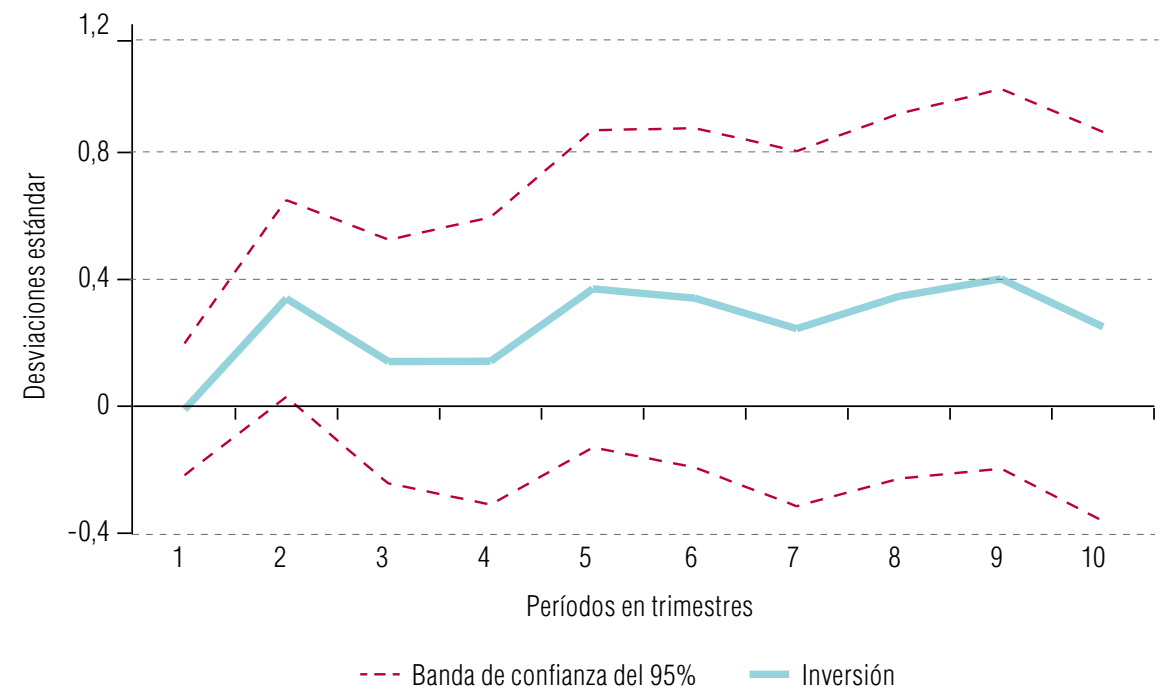

Fuente: Elaboración propia.

En general, se observan respuestas coherentes y comprensibles con base en la teoría económica, pero distantes de los resultados obtenidos por otros autores, en cuanto a la magnitud del impacto. Para el caso de la actividad económica, los resultados permiten inferir que un choque positivo del índice de términos de intercambio empieza a ser significativo a partir del primer trimestre y tiene una duración total de cuatro períodos. Si dicha innovación se da sobre los precios de las exportaciones en mayor proporción, se observa que el incremento de la producción se genera a través de una mejora en los ingresos de los exportadores, lo que permite inferir que son transitorios y no permanentes, como lo ha explicado Parra (2008). En el caso de la inversión se considera que la respuesta es significativa en el segundo trimestre, pero el efecto del choque tiene corta duración, únicamente de un trimestre. Lo anterior se puede explicar por el hecho de que el choque positivo de los términos de intercambio afecta de forma directa y positiva a la producción del país por medio de la balanza comercial, lo que produce incrementos en el ingreso y esto a su vez sobre la inversión en un período posterior. Es decir, la inversión recoge el efecto de los términos de intercambio a partir de las variaciones generadas sobre el nivel de producción o ingreso.

Por su parte, la balanza comercial presenta un efecto significativo y positivo ante la innovación, que dura aproximadamente cinco trimestres desde el primer momento del choque. Este efecto se genera porque el choque positivo de los términos de intercambio incentiva el incremento de la producción nacional de bienes exportados. Por otro lado, al observar el tipo de cambio real se constata que este presenta una respuesta inversa y prolongada (tres trimestres, aproximadamente), lo que se traduce en una apreciación de la moneda nacional frente al dólar debido a la fuerte entrada de divisas extranjeras al país. El efecto de esta apreciación en el corto plazo en los sectores manufactureros depende de la exposición de cada sector a los mercados externos y de la composición de sus insumos (importados o nacionales). Según Sierra y Manrique (2014) y Peláez y Sierra (2016), en el período 2000-2010 la apreciación real del peso pudo afectar negativamente a la mitad del valor agregado industrial (18 sectores) y al 31\% del empleo total (18 sectores). Además, tuvo efectos positivos para el $4 \%$ del valor agregado total (4 sectores) y el $3 \%$ del empleo total manufacturero (7 sectores). Sin embargo, dicha apreciación no surtió un efecto significativo en 38 (34) sectores que forman el $45 \%$ (66\%) del valor agregado total (empleo total) de las manufacturas. 


\section{Gráfico 5}

Impulso-respuesta generalizada y acumulada del factor tipo de cambio real y la balanza comercial ante un choque de una desviación estándar en el índice de términos de intercambio

A. Respuesta acumulada de la balanza comercial ante una innovación del índice de términos de intercambio

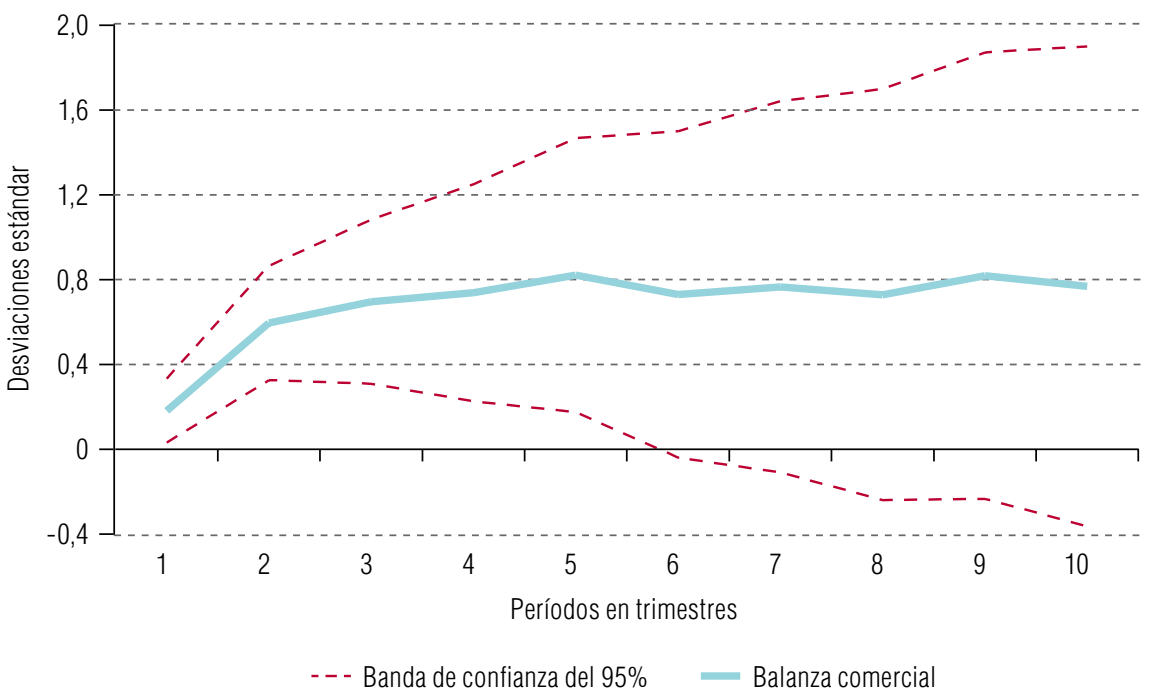

B. Respuesta acumulada del factor tipo de cambio ante una innovación del índice de términos de intercambio

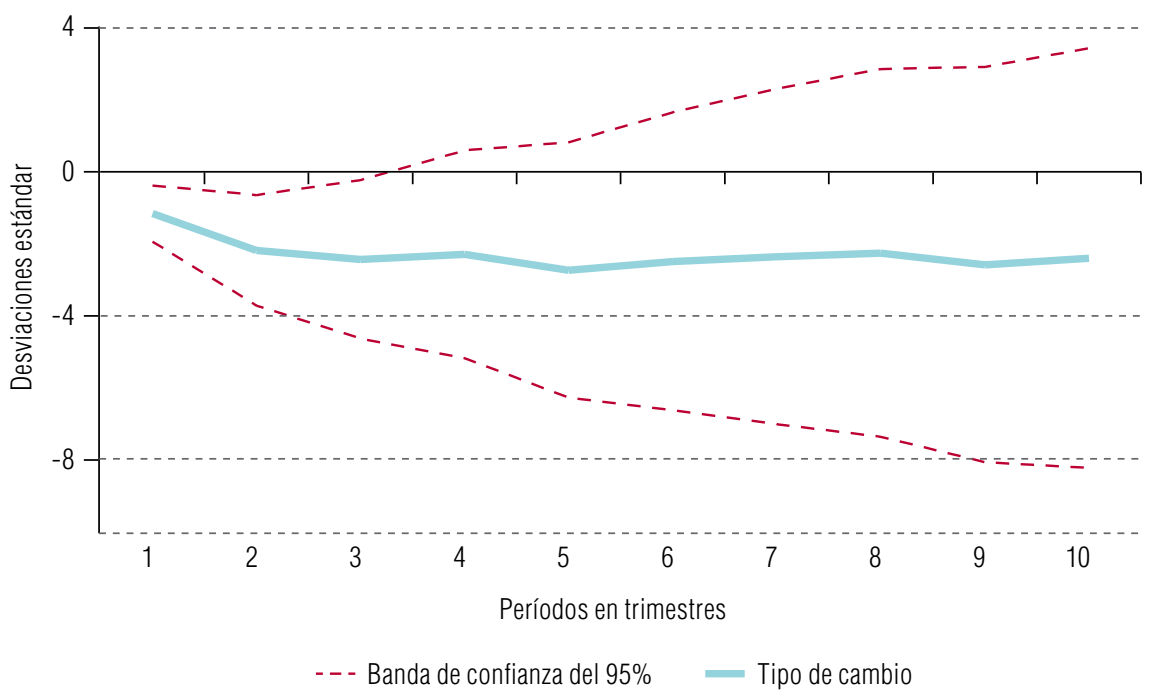

Fuente: Elaboración propia.

Por último, al analizar los factores restantes, los agregados monetarios y precios y la serie del consumo total, se puede concluir que un choque de los términos de intercambio no genera efectos significativos sobre dichas variables. Así lo sostiene Parra (2008), quien encontró que los efectos del choque de los términos de intercambio en la década de 2000 generaron una apreciación de la moneda nacional dada la revaluación del dólar que duró hasta el 2008. Tras analizar el consumo infirió que los choques resultantes de los términos de intercambio no alteraron los niveles de precios agregados. Es decir que, ante el aumento de los precios del petróleo en el período 2003-2014 y dada la fuerte apreciación del peso, ello no dio lugar a un proceso inflacionario. Por ende, es importante resaltar que la inflación no obedece en este caso en particular a choques externos. 
En general, los resultados muestran que efectivamente los términos de intercambio tienen un efecto significativo sobre un conjunto de variables macroeconómicas, que resumen en buena medida el comportamiento de la actividad económica de forma coherente y de conformidad con lo previsto.

\section{Gráfico 6}

Impulso-respuesta generalizada y acumulada del factor agregados monetarios, del factor precios (inflación) y del consumo total ante un choque del índice de términos de intercambio

A. Respuesta acumulada del factor agregados monetarios ante una innovación del índice de términos de intercambio

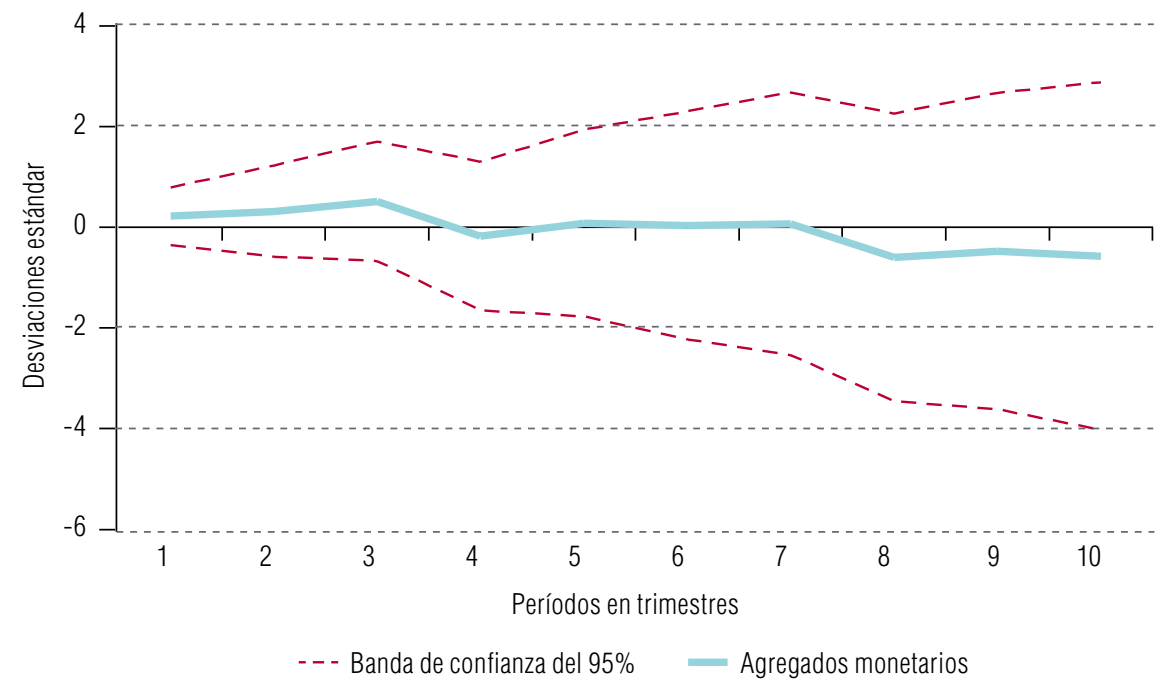

B. Respuesta acumulada del factor de precios ante una innovación del índice de términos de intercambio

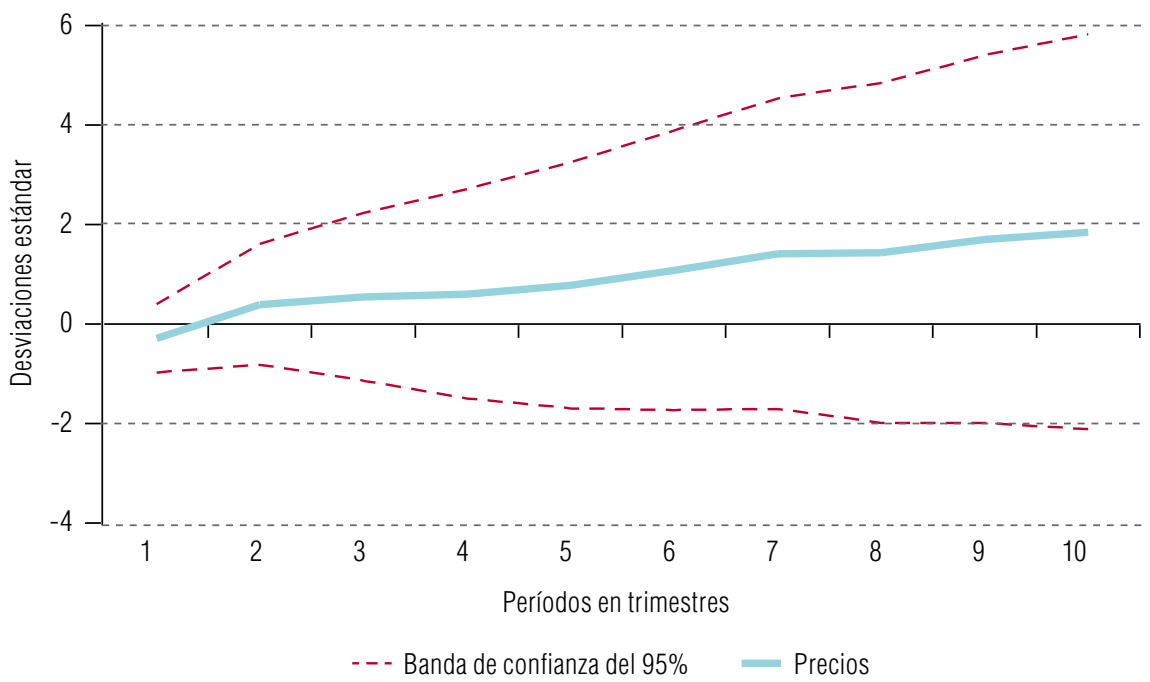


Gráfico 6 (conclusión)

C. Respuesta acumulada del consumo total ante una innovación del índice de términos de intercambio

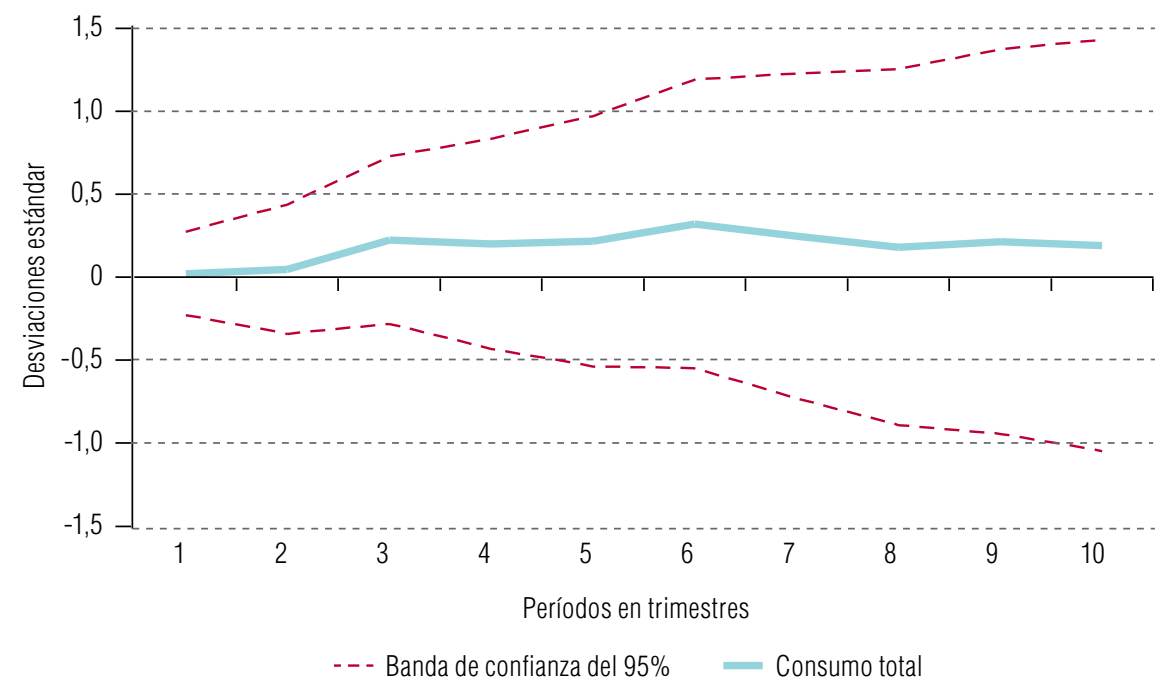

Fuente: Elaboración propia.

\section{Descomposición de varianza}

La descomposición de varianza del error representa la proporción de movimientos de una serie a raíz de sus innovaciones propias en comparación con las de las demás variables del modelo. Es decir, esta metodología logra captar los distintos componentes que permiten aislar el porcentaje de variabilidad de cada una de las variables resultantes de la innovación, con lo que se hace más fácil identificar la dependencia relativa que tiene cada variable sobre el resto. Se utiliza el método de ortogonalización de Cholesky sobre los residuos del FAVAR.

La descomposición de Cholesky supone un ordenamiento de las variables en función de su grado de endogeneidad contemporánea. Las variables se organizan por ende de la más endógena a la más exógena de la siguiente forma: factor de actividad económica, consumo total, inversión, factor de precios, factor de agregados monetarios, balanza comercial, factor de tipo de cambio real e índice de términos de intercambio. Es importante resaltar que en el caso de Colombia damos por sentado que existe exogeneidad en los términos de intercambio, por tres razones: Colombia es una economía relativamente pequeña y abierta al comercio, que no influye en los precios internacionales; tal como se indica en la sección III, existe una alta correlación de los términos de intercambio con el precio del petróleo, determinado a nivel internacional; y por último, cabe señalar que hay un estudio que demuestra la exogeneidad de los términos de intercambio de los países latinoamericanos (véase Ben Zeev, Pappa y Vicondoa, 2016).

Los resultados de la descomposición de varianza para un horizonte finito de 20 trimestres se resumen en el cuadro 3. Se observa que el $5 \%$ de la variabilidad del factor de actividad económica se explica por movimientos en los términos de intercambio. Del mismo modo, el consumo y el factor de agregados monetarios presentan una variabilidad del $5 \%$. Por otra parte, es evidente que la variabilidad de la balanza comercial, el factor de precios y el factor de tipo de cambio real son del $6 \%, 4 \%$ y $1 \%$, respectivamente. Por último, se observa que el $8 \%$ de la variabilidad de la inversión obedece a las variaciones en los términos de intercambio. 
Cuadro 3

Descomposición de la varianza de variables de interés seleccionadas

\begin{tabular}{lc}
\hline Variables & Porcentaje de la varianza que se explica por los términos de intercambio \\
\hline Actividad económica (PIB) & 5,00 \\
\hline Consumo total & 5,00 \\
\hline Agregados monetarios & 5,00 \\
\hline Balanza comercial & 6,00 \\
\hline Precios (inflación) & 4,00 \\
\hline Tipo de cambio real & 1,00 \\
\hline Inversión & 8,00 \\
\hline
\end{tabular}

Fuente: Elaboración propia.

Sin duda la variable que se ve más afectada por un choque en los términos de intercambio es la inversión, que representa una cuarta parte del PIB. La mejora de los términos de intercambio propicia un incremento en la inversión, especialmente en el sector exportador de materias primas, porque la rentabilidad marginal es una función de los precios esperados de las exportaciones. Nuestros resultados no concuerdan con los obtenidos por autores de otros países como Castillo y Salas (2012), quienes afirmaron que los choques en los términos de intercambio explican aproximadamente el $90 \%$ de las fluctuaciones del producto, la inversión y el consumo en un horizonte de 10 años para la economía peruana. Hernández (2013) concluyó que las oscilaciones de la producción colombiana se explican entre un $27 \%$ y un 30\% por los términos de intercambio. Por su parte, Arteaga, Granados y Joya (2013) determinaron que una mejora en los términos de intercambio generaba una caída del $50 \%$ en el tipo de cambio real. Aunque nuestros resultados coinciden con otros estudios relativos a los efectos que sobre las variables tienen los términos de intercambio, la magnitud del efecto es menor.

Para autores como Hernández (2013), Schmitt-Grohé y Uribe (2015) y Broda (2004) existen variaciones significativas en la magnitud del efecto de un choque en los términos de intercambio sobre los diferentes agregados económicos, principalmente por la metodología aplicada. Los modelos basados en simulaciones, como el que elaboró Mendoza (1995), indican que los choques en los términos de intercambio ejercen importantes efectos sobre la actividad económica de los países estudiados, mientras que los modelos VAR revelan efectos idénticos pero de menor cuantía. Se argumenta que dicha desconexión entre ambas metodologías se produce porque se dispone de pocos datos o por la escasa cantidad de rezagos utilizados en los modelos empíricos, es decir que el modelo sufre un sesgo de truncamiento por rezagos (Chari, Kehoe y McGrattan, 2008). Los modelos FAVAR, como alternativa a las metodologías anteriormente mencionadas, tienen como principal ventaja la utilización de una gran cantidad de variables de actividad económica, por lo que captan en mayor medida los efectos indirectos que sobre la actividad económica produce un choque en los términos de intercambio. Según Stock y Watson (2005), los modelos FAVAR reducen la probabilidad de que existan variables omitidas, problema del que adolecen habitualmente los modelos SVAR, por lo que la inclusión de un gran número de series económicas favorece las estimaciones al abarcar la mayoría de los espacios de los choques estructurales de una economía. En este estudio los cuatro factores estimados explican aproximadamente el $60 \%$ de la varianza de las series económicas utilizadas.

\section{Pruebas de robustez}

En la siguiente prueba de robustez se reemplazaron los factores de actividad económica y tipo de cambio real por el PIB (medido en billones de pesos a precios constantes de 2005) y por el índice de tipo de cambio real medido para 18 países miembros del FMI, respectivamente. La estimación 
de las funciones impulso-respuesta se muestra en el gráfico 7 y ello permite inferir que un choque positivo del índice de los términos de intercambio tiene un efecto favorable y significativo sobre el PIB, pero su duración es menor con respecto al factor de actividad económica. Por su parte, en el caso de la inversión se observan dos efectos significativos y positivos, uno en el segundo trimestre y otro en el quinto trimestre, ambos de corta duración. La balanza comercial responde de forma positiva y significativa con mayor duración que en el modelo previo, mientras que el índice de tasa de cambio real se comporta igual que el factor de tipo de cambio real estimado, aunque su respuesta es menos prolongada. Por último, el factor agregados monetarios, el factor precios y la variable de consumo total no sufren alteraciones significativas ante un choque del índice de términos de intercambio, como se evidenció en los resultados anteriores. En resumen, estimadas las funciones impulso-respuesta tras reemplazar los factores de actividad económica y tipo de cambio real por las series correspondientes, se puede inferir que no existen diferencias significativas en relación con el modelo principal descrito.

\section{Gráfico 7}

Impulso-respuesta generalizada y acumulada del PIB, la inversión, la balanza comercial, el índice de tasa de cambio real, los agregados monetarios, los precios y el consumo total

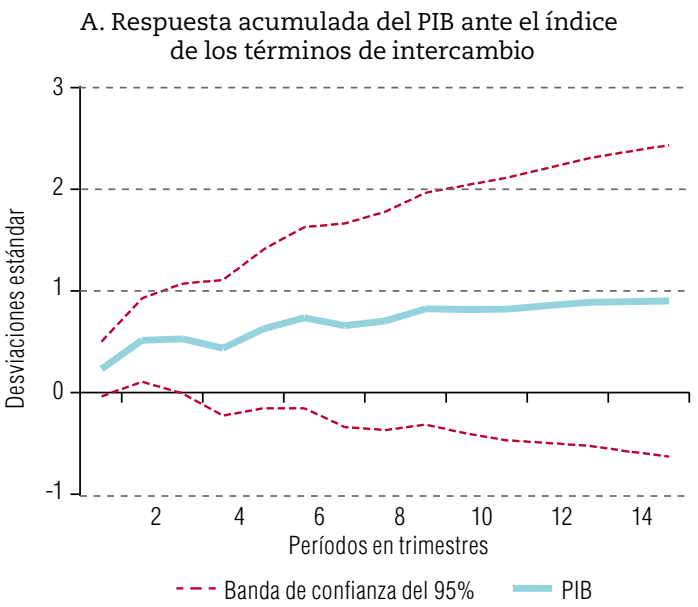

C. Respuesta acumulada de la balanza comercial ante el índice de los términos de intercambio

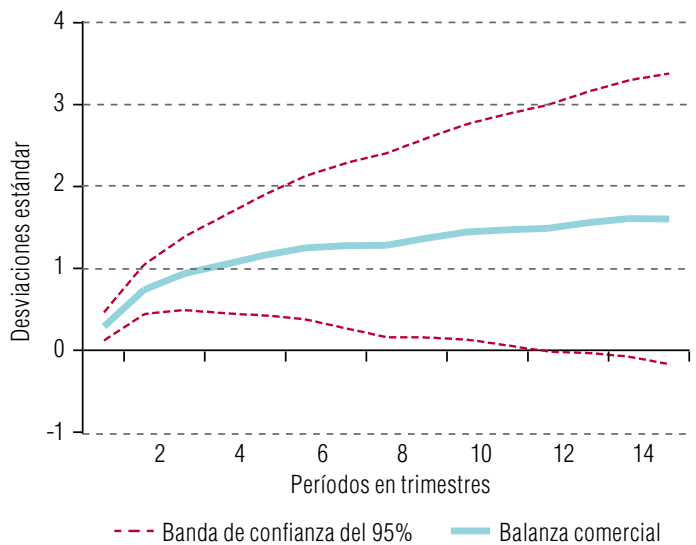

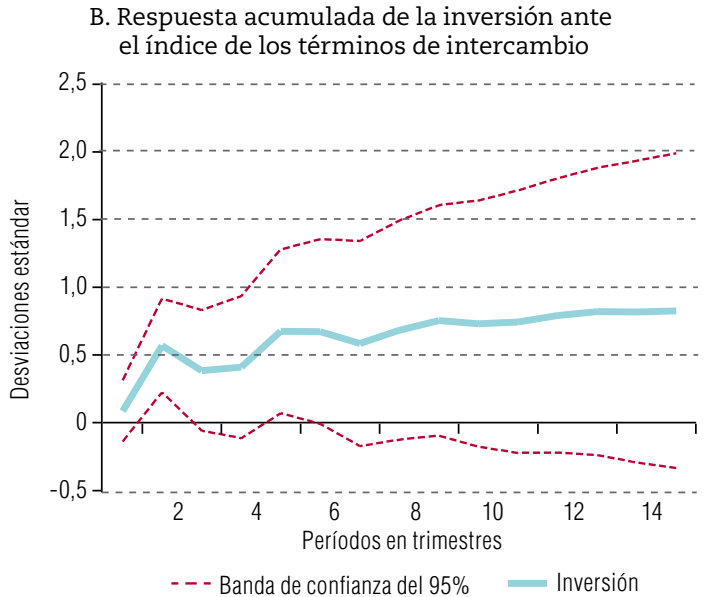

. Respuesta acumulada de la inversión ante el índice de los términos de intercambio

- - - Banda de confianza del 95\% — Inversión

D. Respuesta acumulada del índice de tasa de cambio real ante el índice de los términos de intercambio

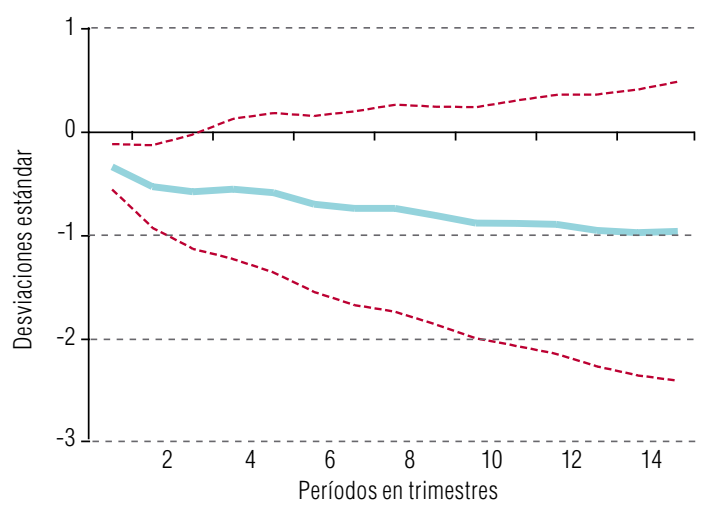

- - - Banda de confianza del 95\% — Tasa de cambio real 
Gráfico 7 (conclusión)

E. Respuesta acumulada del factor agregados monetarios ante el índice de los términos de intercambio

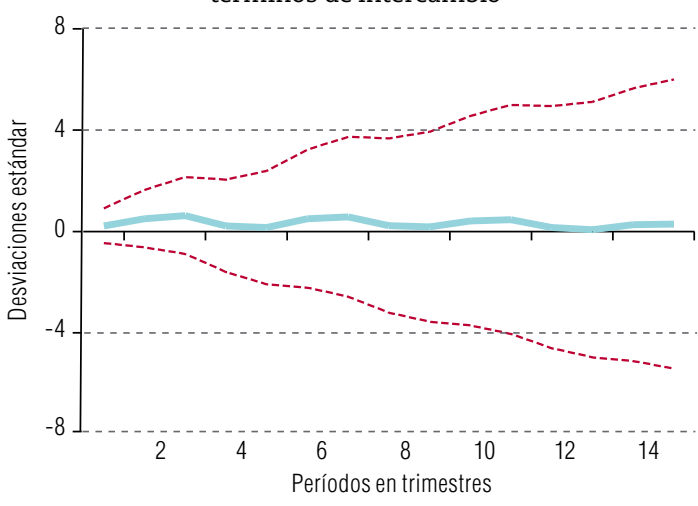

- - - Banda de confianza del 95\% - Agregados monetarios
F. Respuesta acumulada del factor precios ante el índice de los términos de intercambio

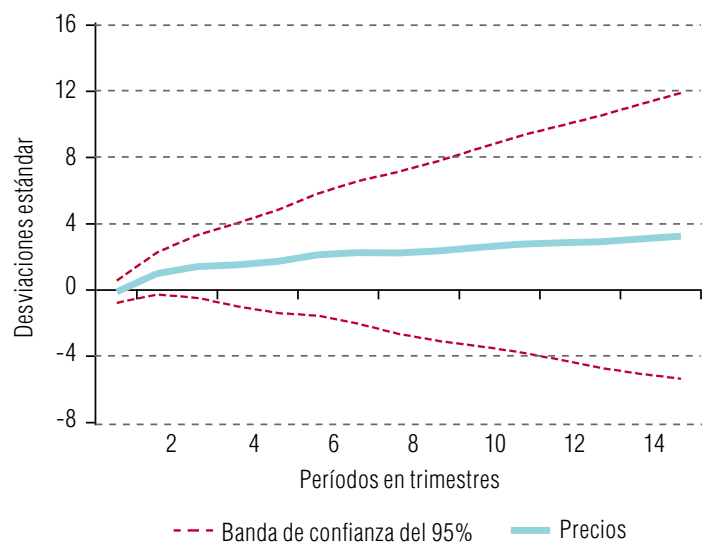

G. Respuesta acumulada del consumo total ante el índice de los términos de intercambio

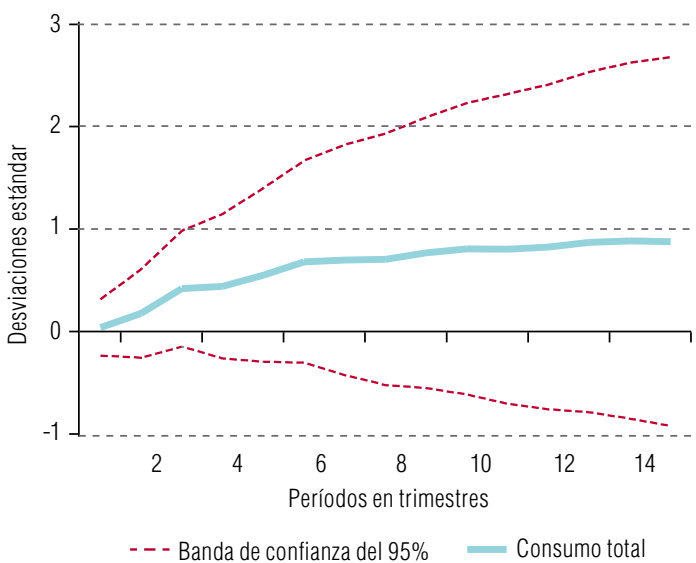

Fuente: Elaboración propia.

Por otro lado, al aplicar la descomposición de varianza se determina que los términos de intercambio explican la variabilidad del PIB en un 6\%, de la inversión en un 9\%, de la balanza comercial en un $10 \%$, del índice de tasa de cambio real en un $2 \%$, del factor agregados monetarios en un $5 \%$, del factor precios en un 5\% y del consumo total en un $4 \%$. Se advierten leves incrementos con respecto a la estimación del modelo de cuatro factores, principalmente en la balanza comercial.

Por último, y con el fin de aislar los efectos de los precios de las exportaciones y las importaciones, se estiman como segunda prueba de robustez las funciones impulso-respuesta de los impulsos positivos generados por los índices de los precios de exportaciones y el de importaciones, respectivamente. Los resultados descritos en el anexo A3 indican que un choque positivo del índice de precios de exportaciones genera efectos positivos y significativos sobre el factor actividad económica, la inversión, la balanza comercial y un efecto negativo y significativo sobre el factor tipo de cambio. Además, se evidencia en la descomposición de varianza (véase el cuadro 1 en la sección $\mathrm{Vl}$ ) que la inversión es la variable que presenta una mayor variación (6\%) ante el choque del índice de términos de intercambio. Por su parte, un choque positivo del índice de precios de importación genera efectos positivos y significativos sobre el factor actividad económica, la inversión, el factor tipo de cambio y el factor precios. Sin embargo, surte importantes 
efectos negativos sobre la balanza comercial. En cuanto a la descomposición de varianza (véase el cuadro 1), se infiere que los precios son los que presentan un mayor efecto (7\%), seguidos por la inversión (5\%). Cabe resaltar que los resultados son similares a los obtenidos gracias a la utilización del modelo principal. Se infiere que el índice de precios de exportaciones genera efectos parecidos al índice de términos de intercambio en cuanto a signo y duración.

\section{Conclusiones}

Este artículo tiene por objeto analizar la incidencia de los choques en los términos de intercambio sobre la economía colombiana, que concentra gran parte de sus exportaciones en materias primas. Se tomó como base el hecho de que los estudios previos no habían analizado la persistencia y la influencia de los choques exógenos resultantes de las variaciones de los precios de bienes exportados e importados sobre los diferentes agregados que componen la economía. Por lo tanto, se hizo uso de un elevado número de series trimestrales para el período 2001-2016, con el fin de abarcar gran parte de los diferentes sectores económicos de Colombia y de esa forma aprovechar las ventajas que ofrece la metodología FAVAR para explicar de forma más detallada la transmisión de esas innovaciones a la economía.

Los resultados obtenidos muestran que los choques en los términos de intercambio generan un efecto significativo sobre la actividad económica, la inversión, el tipo de cambio real y la balanza comercial. Cabe tener en cuenta que las características de los productos exportados y el nivel de apertura de la economía juegan un papel importante en la determinación del impacto, pero para el caso de Colombia es posible afirmar que los términos de intercambio influyen de forma relevante sobre las variables económicas descritas, especialmente sobre la inversión. La implementación de un modelo FAVAR garantiza el análisis de funciones impulso-respuesta de las series macroeconómicas de interés, con lo que se pudo observar que un choque positivo produce un auge de cuatro períodos sobre la actividad económica, una apreciación de tres períodos sobre el tipo de cambio real y un auge de seis períodos sobre la balanza comercial. En el caso de la inversión se registra un incremento que dura menos de un trimestre. Por otro lado, tras el análisis de descomposición de varianza surgen datos que muestran que para los 20 períodos posteriores al choque de los términos de intercambio, estos últimos explican la variabilidad de la inversión en un 8\%, de la balanza comercial en un 6\%, de la actividad económica en un $5 \%$ y del tipo de cambio real en un $1 \%$.

\section{Bibliografía}

Aguirre, E. (2011), "Essays on exchange rates and emerging markets", tesis de doctorado, Nueva York, Universidad de Columbia.

Ahmed, S. (2003), "Sources of economic fluctuations in Latin America and implications for choice of exchange rate regimes", Journal of Development Economics, vol. 72, № 1, Amsterdam, Elsevier.

Andrews, D. y D. Rees (2009), "Macroeconomic volatility and terms of trade shocks", Research Discussion Paper, N ${ }^{\circ}$ 2009-05, Reserve Bank of Australia (RBA).

Arteaga, C., J. Granados y J. Joya (2013), "El comportamiento del tipo de cambio real en Colombia: ¿explicado por sus fundamentales?", Ensayos sobre Política Económica, vol. 31, № 72, Amsterdam, Elsevier.

Bai, J. y S. Ng (2002), "Determining the number of factors in approximate factor models", Econometrica, vol. 70, № 1, Hoboken, Wiley.

Banco Mundial (2016), "Exportaciones de bienes y servicios (\% del PIB)" [en línea] https://data.worldbank. org/indicator/NE.EXP.GNFS.ZS?locations=CO.

(2000), Securing our Future in a Global Economy, Washington, D.C. 
Ben Zeev, N., E. Pappa y A. Vicondoa (2016), "Emerging economies business cycles: the role of the terms of trade revisited", Working Paper, № 1610, Beerseba, Ben-Gurion University of the Negev.

Bernanke, B., J. Boivin y P. Eliasz (2005), "Measuring the effects of monetary policy: a factor-augmented vector autoregressive (FAVAR) approach", The Quarterly Journal of Economics, vol. 120, № 1, Oxford, Oxford University Press.

Broda, C. (2004), "Terms of trade and exchange rate regimes in developing countries", Journal of International Economics, vol. 63, № 1, Amsterdam, Elsevier.

Cano, G. y otros (2012), "El mercado mundial del café y su impacto en Colombia", Borradores de Economía, № 710, Bogotá, Banco de la República.

Castillo, P. y J. Salas (2012), Los términos de intercambio como impulsores de fluctuaciones económicas en economías en desarrollo: estudio empírico, Ciudad de México, Centro de Estudios Monetarios Latinoamericanos (CEMLA).

Chari, V., P. Kehoe y E. McGrattan (2008), "Are structural VARs with long-run restrictions useful in developing business cycle theory?", Journal of Monetary Economics, vol. 55, № 8, Amsterdam, Elsevier.

Charnavoki, V. y J. Dolado (2014), "The effects of global shocks on small commodity-exporting economies: lessons from Canada", American Economic Journal: Macroeconomics, vol. 6, № 2, Nashville, American Economic Association.

Dickey, D. y W. Fuller (1979), "Distribution of the estimators for autoregressive time series with a unit root", Journal of the American Statistical Association, vol. 74, № 366a, Abingdon, Taylor \& Francis.

Fernández, A., A. González y D. Rodríguez (2015), "Sharing a ride on the commodities roller coaster: common factors in business cycles of emerging economies", Borradores de Economía, № 915, Bogotá, Banco de la República.

Forni, M. y otros (2005), "The generalized dynamic factor model: one-sided estimation and forecasting", Journal of the American Statistical Association, vol. 100, № 471, Abingdon, Taylor \& Francis. (2000), "The generalized dynamic-factor model: identification and estimation", The Review of Economics and Statistics, vol. 82, N4 , Cambridge, MIT Press.

Garavito, A. y otros (2011), "Construcción del índice de términos de intercambio para Colombia", Borradores de Economía, № 639, Bogotá, Banco de la República.

Gaviria, A. (1993), "El ahorro privado y los términos de intercambio: el caso colombiano", Ensayos sobre Política Económica, vol. 12, № 23, Bogotá, Banco de la República.

Geweke, J. (1978), The Dynamic Factor Analysis of Economic Time Series Models, Universidad de Wisconsin.

Gómez, V. y A. Maravall (1998), "Seasonal adjustment and signal extraction in economic time series", Documento de Trabajo, № 9809, Madrid, Banco de España.

Hernández, G. (2013), "Colombia: términos de intercambio y fluctuaciones de la producción", Revista CEPAL, № 110 (LC/G.2572-P), Comisión Económica para América Latina y el Caribe (CEPAL).

Izquierdo, A., R. Romero y E. Talvi (2008), "Booms and busts in Latin America: the role of external factors", Working Paper, № 631, Washington, D.C., Banco Interamericano de Desarrollo (BID).

Jääskelä, J. y P. Smith (2013), "Terms of trade shocks: what are they and what do they do?", Economic Record, vol. 89, № 285, Hoboken, Wiley.

Jenkins, R. (2011), "El 'efecto China' en los precios de los productos básicos y en el valor de las exportaciones de América Latina", Revista CEPAL, № 103 (LC/G.2487-P), Comisión Económica para América Latina y el Caribe (CEPAL).

Karagedikli, O. y G. Price (2013), "Identifying terms of trade shocks and their transmission to the New Zealand economy" [en línea] http://docs.business.auckland.ac.nz/Doc/Paper-10_Gael.pdf.

Mendoza, E. (1995), "The terms of trade, the real exchange rate, and economic fluctuations", International Economic Review, vol. 36, № 1, Hoboken, Wiley.

Mumtaz, H. y P. Surico (2009), "The transmission of international shocks: a factor-augmented VAR approach", Journal of Money, Credit and Banking, vol. 41, № s1, Hoboken, Wiley.

Ortiz, C. (2016), Diversificación productiva y crecimiento económico: enfoques teóricos y análisis del desarrollo socio-económico de Colombia, Cali, Universidad del Valle.

Parra, J. (2008), "Hechos estilizados de la economía colombiana: fundamentos empíricos para la construcción y evaluación de un modelo DSGE", Borradores de Economía, №509, Bogotá, Banco de la República.

Peláez, J. y L. Sierra (2016), "Does industrial employment react to movements in the real exchange rate? An empirical analysis for Colombia, 2000-2010", Latin American Journal of Economics, vol. 53, № 1, Santiago, Pontificia Universidad Católica de Chile. 
Pesaran, H. e Y. Shin (1998), "Generalized impulse response analysis in linear multivariate models", Economics Letters, vol. 58, No 1, Amsterdam, Elsevier.

Phillips, P. y P. Perron (1988), "Testing for a unit root in time series regression", Biometrika, vol. 75, № 2, Oxford, Oxford University Press.

Poncela, P., E. Senra y L. Sierra (2017), "Long-term links between raw materials prices, real exchange and relative de-industrialization in a commodity-dependent economy: empirical evidence of 'Dutch disease' in Colombia", Empirical Economics, vol. 52, № 2, Nueva York, Springer.

Roberts, M. y W. Schlenker (2013), "Identifying supply and demand elasticities of agricultural commodities: implications for the US ethanol mandate", The American Economic Review, vol. 103, № 6, Nashville, American Economic Association.

Schmitt-Grohé, S. y M. Uribe (2015), "How important are terms of trade shocks?", NBER Working Paper, $N^{\circ} 21253$, Cambridge, Massachusetts, National Bureau of Economic Research (NBER).

Sierra, L. (2015), “¿Qué tanto afecta Asia a Latinoamérica?: el canal de las materias primas”, Las relaciones económicas entre América Latina y Asia: hacia la construcción de una nueva inserción internacional, M. de Miranda y J. Peláez (comps.), Cali, Pontificia Universidad Javeriana de Cali.

Sierra, L. y L. Manrique (2014), "Impacto del tipo de cambio real en los sectores industriales de Colombia: una primera aproximación", Revista CEPAL, № 114 (LC/G.2629-P), Santiago, Comisión Económica para América Latina y el Caribe (CEPAL).

Sinnott, E., J. Nash y A. de la Torre (2010), Natural resources in Latin America and the Caribbean: beyond booms and busts?, Washington, D.C., Banco Mundial.

Spatafora, N. y A. Warner (1999), "Macroeconomic and sectoral effects of terms-of-trade shocks: the experience of the oil-exporting developing countries", IMF Working Paper, No 99/134, Washington, D.C, Fondo Monetario Internacional (FMI).

Stock, J. y M. Watson (2011), "Dynamic factor models", Oxford Handbook of Economic Forecasting, M. Clements y D. Hendry (eds.), Oxford, Oxford University Press.

(2005), "Implications of dynamic factor models for VAR analysis", NBER Working Paper, N 11467 , Cambridge, Massachusetts, National Bureau of Economic Research (NBER).

(2002), "Macroeconomic forecasting using diffusion indexes", Journal of Business \& Economic Statistics, vol. 20, N 2, Abingdon, Taylor \& Francis. 


\section{Anexo A1}

Las series fueron tomadas del Banco de la República (BR), del Departamento Administrativo Nacional de Estadística (DANE), del Ministerio de Hacienda y Crédito Público (MH) y del Fondo Monetario Internacional (FMI). Las trasformaciones de cada serie se enuncian de la siguiente forma: 1 - Sin transformación; 2 - Logaritmo; 3 - Primera diferencia; 4 - Primera diferencia del logaritmo.

Cuadro A1.1

Descripción de las variables de interés

\begin{tabular}{clcc}
\hline Número & \multicolumn{1}{c}{ Variable } & Fuente & Transformación \\
\hline 1 & Índice términos de intercambio & BR & 4 \\
\hline 2 & PIB & DANE & 4 \\
\hline 3 & Consumo total & DANE & 4 \\
\hline 4 & Formación bruta de capital fijo (inversión) & DANE & 4 \\
\hline 5 & Balanza comercial (en miles de millones de pesos) & BR & 4 \\
\hline 6 & Tipo de cambio respecto a 18 países miembros del FMl & BR & 4 \\
\hline 7 & Inflación total & BR & 4 \\
\hline
\end{tabular}

Fuente: Elaboración propia, sobre la base de datos de Banco de la República (BR), Departamento Administrativo Nacional de Estadística (DANE), Ministerio de Hacienda y Crédito Público y Fondo Monetario Internacional (FMI).

Cuadro A1.2

Descripción de las 129 series utilizadas

\begin{tabular}{|c|c|c|c|}
\hline Número & Variable & Fuente & Transformación \\
\hline \multicolumn{4}{|c|}{ Actividad económica a precios constantes (en miles de millones de pesos) } \\
\hline 1 & Crecimiento PIB & DANE & 1 \\
\hline 2 & Índice de producción real de la industria manufacturera & DANE & 4 \\
\hline 3 & Producción de bienes de la industria: cemento gris (toneladas) & DANE & 4 \\
\hline 4 & Producción de bienes de la industria: vehículos ensamblados (cantidad) & DANE & 4 \\
\hline 5 & Producción de bienes de la industria: azúcar (toneladas) & DANE & 2 \\
\hline 6 & Consumo final del Gobierno & DANE & 2 \\
\hline 7 & Consumo final de los hogares & DANE & 2 \\
\hline 8 & Consumo de bienes duraderos (porcentaje) & DANE & 1 \\
\hline 9 & Consumo de bienes no duraderos (porcentaje) & DANE & 1 \\
\hline 10 & Consumo de bienes semiduraderos (porcentaje) & DANE & 1 \\
\hline 11 & Consumo de servicios (porcentaje) & DANE & 1 \\
\hline 12 & Demanda final interna & DANE & 4 \\
\hline 13 & Formación bruta de capital & DANE & 4 \\
\hline 14 & Formación bruta de capital: agropecuario, silvicultura, caza y pesca & DANE & 1 \\
\hline 15 & Formación bruta de capital: construcción & DANE & 1 \\
\hline 16 & Formación bruta de capital: equipo de transporte & DANE & 1 \\
\hline 17 & Formación bruta de capital: maquinaria y equipo & DANE & 1 \\
\hline 18 & Formación bruta de capital: obras civiles & DANE & 1 \\
\hline 19 & Formación bruta de capital: servicios & DANE & 1 \\
\hline 20 & Variaciones de las existencias & DANE & 1 \\
\hline 21 & Deuda interna total & $\mathrm{MH}$ & 4 \\
\hline 22 & Deuda interna: desembolsos & $\mathrm{MH}$ & 4 \\
\hline 23 & Deuda interna: amortización & $\mathrm{MH}$ & 2 \\
\hline
\end{tabular}


Cuadro A1.2 (continuación)

\begin{tabular}{|c|c|c|c|}
\hline Número & Variable & Fuente & Transformación \\
\hline \multicolumn{4}{|c|}{ Sector externo (en miles de millones de pesos) } \\
\hline 24 & Deuda externa & $\mathrm{MH}$ & 4 \\
\hline 25 & Exportaciones totales & DANE & 4 \\
\hline 26 & Exportaciones de café & DANE & 4 \\
\hline 27 & Exportaciones de café (toneladas métricas) & DANE & 4 \\
\hline 28 & Exportaciones de carbón & DANE & 4 \\
\hline 29 & Exportaciones de carbón (toneladas métricas) & DANE & 2 \\
\hline 30 & Exportaciones de níquel & DANE & 4 \\
\hline 31 & Exportaciones de níquel (toneladas métricas) & DANE & 2 \\
\hline 32 & Exportaciones de petróleo y derivados & DANE & 4 \\
\hline 33 & Exportaciones de petróleo y derivados (toneladas métricas) & DANE & 4 \\
\hline 34 & Exportaciones totales tradicionales & DANE & 4 \\
\hline 35 & Exportaciones totales no tradicionales & DANE & 4 \\
\hline 36 & Importaciones totales & DANE & 4 \\
\hline 37 & Importaciones de bienes intermedios y materias primas: sector agrícola & DANE & 4 \\
\hline 38 & Importaciones de bienes intermedios y materias primas: sector industrial & DANE & 4 \\
\hline 39 & Importaciones de bienes intermedios y materias primas: combustibles & DANE & 4 \\
\hline 40 & Importaciones de bienes de capital: sector agrícola & DANE & 4 \\
\hline 41 & Importaciones de bienes de capital: sector industrial & DANE & 4 \\
\hline 42 & Importaciones de bienes de capital: equipos de transporte & DANE & 4 \\
\hline 43 & Importaciones de bienes de capital: materiales de construcciones & DANE & 4 \\
\hline 44 & Importaciones de bienes de consumo: duraderos & DANE & 4 \\
\hline 45 & Importaciones de bienes de consumo: no duraderos & DANE & 4 \\
\hline 46 & Índice de importaciones de bienes de valor & $\mathrm{FMl}$ & 4 \\
\hline \multicolumn{4}{|c|}{ Tipo de cambio real } \\
\hline 47 & Tipo de cambio comercio total: datos de comercio exterior & $\mathrm{BR}$ & 4 \\
\hline 48 & Tipo de cambio comercio total: datos de índice de precios del productor & $\mathrm{BR}$ & 4 \\
\hline 49 & Tipo de cambio no tradicional: datos de comercio exterior & $\mathrm{BR}$ & 4 \\
\hline 50 & Tipo de cambio no tradicional: datos del índice de precios al productor & BR & 4 \\
\hline 51 & Tipo de cambio indicador competitividad en el mercado de los Estados Unidos & $\mathrm{BR}$ & 4 \\
\hline \multicolumn{4}{|c|}{ Precios: índice de precios al consumidor (IPC) } \\
\hline 52 & Total & DANE & 4 \\
\hline 53 & Alimentos & DANE & 3 \\
\hline 54 & Comunicaciones & DANE & 3 \\
\hline 55 & Diversión & DANE & 3 \\
\hline 56 & Vestuario & DANE & 3 \\
\hline 57 & Educación & DANE & 4 \\
\hline 58 & Otros gastos & DANE & 4 \\
\hline 59 & Salud & DANE & 4 \\
\hline 60 & Transporte & DANE & 4 \\
\hline 61 & Vivienda & DANE & 4 \\
\hline 62 & Variación anual & $\mathrm{BR}$ & 3 \\
\hline 63 & Bienes regulados & $\mathrm{BR}$ & 4 \\
\hline
\end{tabular}


Cuadro A1.2 (continuación)

\begin{tabular}{|c|c|c|c|}
\hline Número & Variable & Fuente & Transformación \\
\hline 64 & Bienes regulados: variación anual & BR & 4 \\
\hline 65 & Bienes transables & $\mathrm{BR}$ & 4 \\
\hline 66 & Bienes no transables & BR & 4 \\
\hline 67 & Bienes no transables: variación anual & BR & 4 \\
\hline \multicolumn{4}{|c|}{ Precios: índice de precios al productor (IPP) } \\
\hline 68 & Clasificación Cuode: bienes de capital & DANE & 4 \\
\hline 69 & Clasificación Cuode: consumo final & DANE & 4 \\
\hline 70 & Clasificación Cuode: consumo intermedio & DANE & 4 \\
\hline 71 & Clasificación Cuode: materiales de construcción & DANE & 4 \\
\hline 72 & Oferta interna & DANE & 4 \\
\hline 73 & Oferta interna: agricultura, ganadería, caza, silvicultura y pesca & DANE & 4 \\
\hline 74 & Oferta interna: industria & DANE & 4 \\
\hline 75 & Oferta interna: minería & DANE & 4 \\
\hline 76 & Producción nacional & DANE & 4 \\
\hline 77 & Producción nacional: agricultura, ganadería, caza, silvicultura y pesca & DANE & 4 \\
\hline 78 & Producción nacional: industria & DANE & 4 \\
\hline 79 & Producción nacional: minería & DANE & 4 \\
\hline 80 & Procedencias: producidos y consumidos & DANE & 4 \\
\hline 81 & Procedencias: exportados & DANE & 4 \\
\hline 82 & Procedencias: importados & DANE & 4 \\
\hline \multicolumn{4}{|c|}{ Agregados monetarios (en miles de millones de pesos) } \\
\hline 83 & Base monetaria: efectivo & $\mathrm{BR}$ & 4 \\
\hline 84 & Base monetaria: reserva bancaria & $\mathrm{BR}$ & 4 \\
\hline 85 & Base monetaria total & $\mathrm{BR}$ & 4 \\
\hline 86 & Bonos & $\mathrm{BR}$ & 4 \\
\hline 87 & Cuentas corrientes: sector privado & $\mathrm{BR}$ & 4 \\
\hline 88 & Cuentas corrientes: sector público & BR & 4 \\
\hline 89 & Cuentas corrientes: total & $\mathrm{BR}$ & 4 \\
\hline 90 & Cuasidineros: cuentas de ahorro & $\mathrm{BR}$ & 4 \\
\hline 91 & Cuasidineros: Certificados de Depósito a Término Fijo (CDT) & $\mathrm{BR}$ & 2 \\
\hline 92 & Cuasidineros: total & $\mathrm{BR}$ & 4 \\
\hline 93 & Depósitos fiduciarios & $\mathrm{BR}$ & 4 \\
\hline 94 & Depósitos a la vista & $\mathrm{BR}$ & 4 \\
\hline 95 & M1 & $B R$ & 4 \\
\hline 96 & M2 & $\mathrm{BR}$ & 4 \\
\hline 97 & M3 & $\mathrm{BR}$ & 4 \\
\hline 98 & $\begin{array}{l}\text { Depósitos en cuenta corriente + cuasidineros + bonos + depósitos a la vista } \\
+ \text { repos + depósitos fiduciarios + certificados de } \\
\text { depósito: total de pasivos sujetos a encaje }\end{array}$ & $\mathrm{BR}$ & 4 \\
\hline \multicolumn{4}{|c|}{ Empleo total nacional } \\
\hline 99 & Desocupados (miles) & DANE & 4 \\
\hline 100 & Inactivos (miles) & DANE & 4 \\
\hline 101 & Ocupados (miles) & DANE & 4 \\
\hline 102 & Tasa de desempleo & DANE & 4 \\
\hline 103 & Tasa de participación & DANE & 4 \\
\hline 104 & Tasa de ocupación & DANE & 4 \\
\hline
\end{tabular}


Cuadro A1.2 (conclusión)

\begin{tabular}{|c|c|c|c|}
\hline Número & Variable & Fuente & Transformación \\
\hline \multicolumn{4}{|c|}{ Sector público no financiero (en miles de millones de pesos) } \\
\hline 105 & Déficit o superávit & $\mathrm{MH}$ & 3 \\
\hline 106 & Financiamiento interno & $\mathrm{MH}$ & 3 \\
\hline 107 & Déficit o superávit empresas públicas no financieras & $\mathrm{MH}$ & 1 \\
\hline 108 & Gastos & $\mathrm{MH}$ & 4 \\
\hline 109 & Ingresos & $\mathrm{MH}$ & 4 \\
\hline 110 & Intereses & $\mathrm{MH}$ & 4 \\
\hline 111 & Financiamiento externo & $\mathrm{MH}$ & 1 \\
\hline \multicolumn{4}{|c|}{ Flujos de inversión extranjera en Colombia (en miles de millones de pesos) } \\
\hline 112 & Sector petrolero & $\mathrm{BR}$ & 3 \\
\hline 113 & Manufacturero & $\mathrm{BR}$ & 4 \\
\hline 114 & Flujos de inversión extranjera total & $\mathrm{BR}$ & 4 \\
\hline 115 & Agricultura, caza, silvicultura y pesca & $\mathrm{BR}$ & 1 \\
\hline 116 & Comercio, restaurantes y hoteles & $\mathrm{BR}$ & 1 \\
\hline 117 & Construcción & $\mathrm{BR}$ & 1 \\
\hline 118 & Electricidad, gas y agua & $\mathrm{BR}$ & 1 \\
\hline 119 & Minas y canteras & $\mathrm{BR}$ & 1 \\
\hline 120 & Servicios financieros y empresariales & $\mathrm{BR}$ & 1 \\
\hline 121 & Servicios comunales & $\mathrm{BR}$ & 1 \\
\hline 122 & Transporte, almacenamiento y comunicaciones & $\mathrm{BR}$ & 1 \\
\hline 123 & Subtotal resto de sectores & $\mathrm{BR}$ & 2 \\
\hline \multicolumn{4}{|c|}{ Tasas de interés } \\
\hline 124 & Tasa de captación mensual & $\mathrm{BR}$ & 3 \\
\hline 125 & Tasa de interés externa: tasa preferencial & $\mathrm{BR}$ & 3 \\
\hline 126 & Tasa de interés de colocación del Banco República & $\mathrm{BR}$ & 1 \\
\hline 127 & Tasa de interés de colocación total & $\mathrm{BR}$ & 1 \\
\hline 128 & Tasa de intervención del Banco República & $\mathrm{BR}$ & 1 \\
\hline 129 & Tasa interés de colocación sin tesorería & $\mathrm{BR}$ & 1 \\
\hline
\end{tabular}

Fuente: Elaboración propia, sobre la base de datos de Banco de la República, Departamento Administrativo Nacional de Estadística (DANE), Ministerio de Hacienda y Crédito Público y Fondo Monetario Internacional (FMI). 


\section{Anexo A2}

El cuadro A2.1 contiene la información sobre el número de rezagos según el criterio de selección. Se utilizaron tres rezagos dado que para este valor el modelo es robusto y consistente en la evaluación de los supuestos sobre los residuales.

\section{Cuadro A2.1}

Criterios de selección de rezagos

\begin{tabular}{lccccc}
\hline Criterio & LR & FPE & AlC & SC & HQ \\
\hline Número de rezagos & 4 & 4 & 4 & 0 & 1 \\
\hline
\end{tabular}

Fuente: Elaboración propia.

Nota: LR: prueba de razón de verosimilitud; FPE: criterio de error de previsión final; AIC: criterio de información Akaike; SC: criterio de información Schwarz; HQ: criterio de información Hannan-Quinn.

El cuadro A2.2 muestra la prueba de auto-correlación (prueba LM) sobre los residuales.

Cuadro A2.2

Prueba de autocorrelación (test de multiplicador de Lagrange)

\begin{tabular}{ccl}
\hline Período & Estadístico de Lagrange (LM-Stat) & p-valor \\
\hline 1 & 79,74127 & 0,0886 \\
\hline 2 & 75,90989 & 0,1464 \\
\hline 3 & 69,67821 & 0,2924 \\
\hline 4 & 66,85996 & 0,3791 \\
\hline 5 & 67,29289 & 0,3651 \\
\hline 6 & 47,04699 & 0,9447 \\
\hline 7 & 74,02127 & 0,1836
\end{tabular}

Fuente: Elaboración propia.

Nota: Hipótesis nula: no existe correlación en los residuales. 


\section{Anexo A3}

Gráfico A3.1

Funciones impulso-respuesta generalizadas y acumuladas de actividad económica, inversión, balanza comercial, factor tipo de cambio, factor precios, factor agregados monetarios y consumo total, respuesta generalizada de una desviación estándar en el índice de precios de exportaciones

\section{Respuesta acumulada de una desviación estándar de las innovaciones de Cholesky con un error estándar de \pm 2}

A. Respuesta del factor actividad económica ante el índice de precios de exportaciones

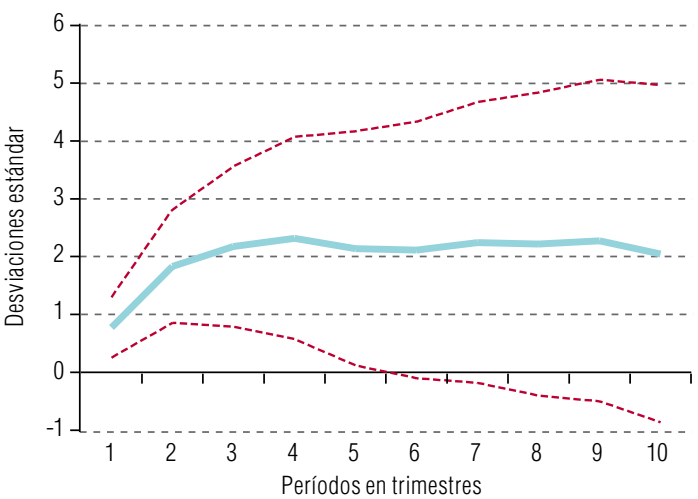

- - - Banda de confianza del 95\% — Actividad económica

C. Respuesta de la balanza comercial ante el índice de precios de exportaciones

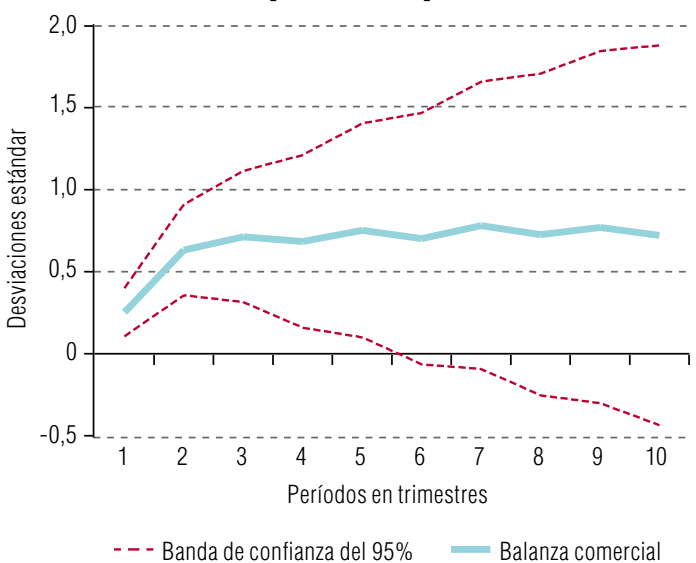

B. Respuesta de la inversión ante el índice de precios de exportaciones

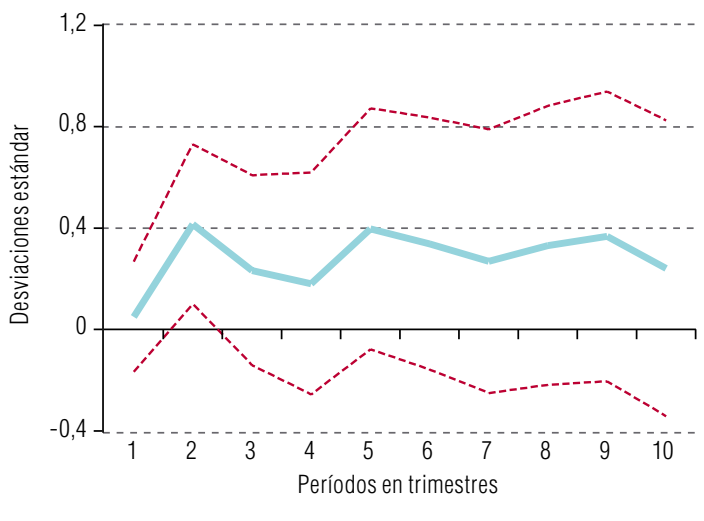

- - - Banda de confianza del 95\% — Inversión

D. Respuesta del factor tipo de cambio ante el índice de precios de exportaciones

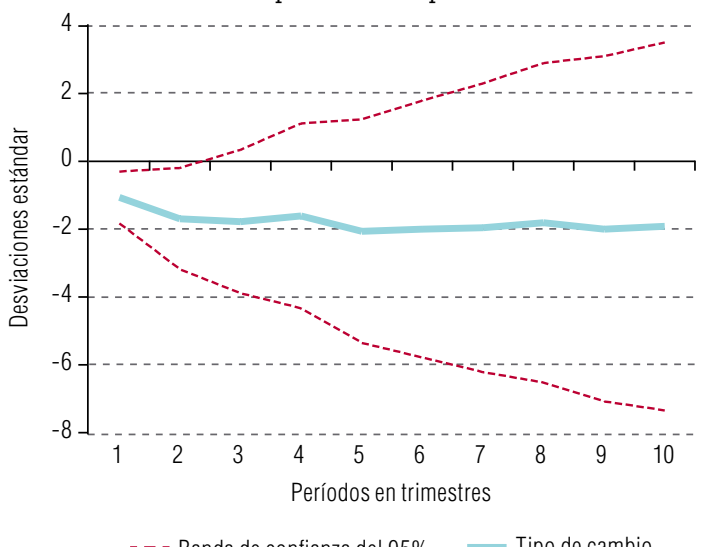


Cuadro A3.1 (conclusión)

E. Respuesta del factor agregados monetarios ante el índice de precios de exportaciones

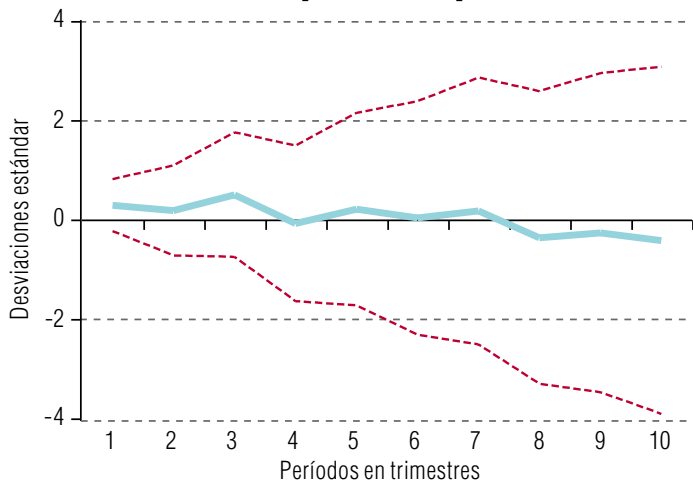

- - - Banda de confianza del 95\% $=$ Agregados monetarios
F. Respuesta del factor precios ante el índice de precios de exportaciones

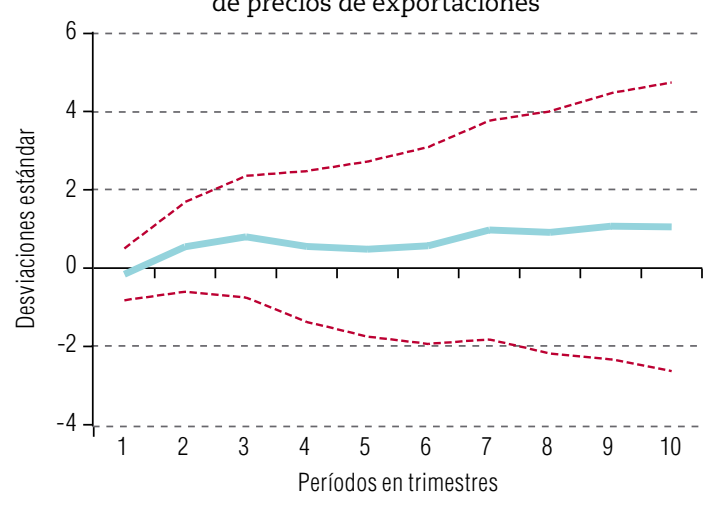

- - - Banda de confianza del 95\% Precios

G. Respuesta del consumo total ante el índice de precios de exportaciones

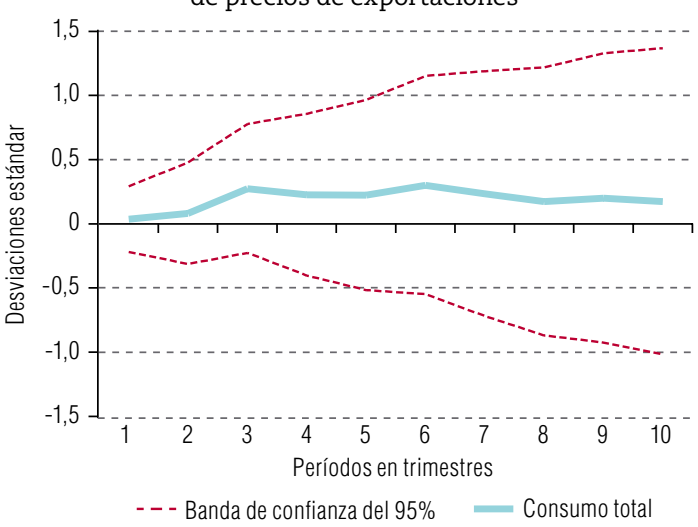

Fuente: Elaboración propia.

\section{Gráfico A3.2}

Funciones impulso-respuesta generalizadas y acumuladas de actividad económica, inversión, balanza comercial, tipo de cambio, precios, agregados monetarios y consumo total, respuesta generalizada de una desviación estándar en el índice de precios de importaciones

\section{Respuesta acumulada de una desviación estándar de las innovaciones de Cholesky con un error estándar de \pm 2}

A. Respuesta del factor actividad económica ante el índice de precios de importaciones

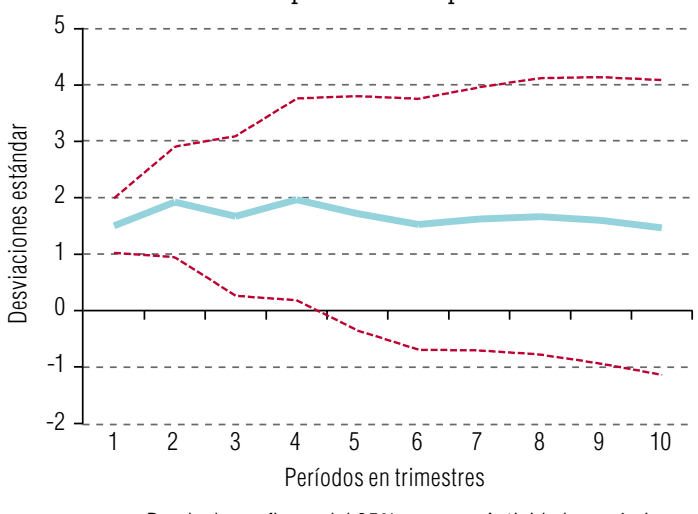

- - - Banda de confianza del 95\% - Actividad económica
B. Respuesta de la inversión ante el índice de precios de importaciones

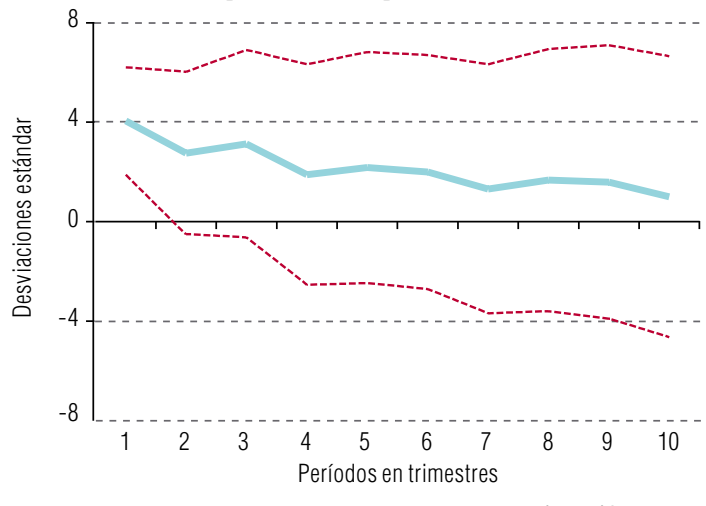

- - - Banda de confianza del 95\% — Inversión 
Cuadro A3.2 (conclusión)

C. Respuesta de la balanza comercial ante el índice de precios de importaciones

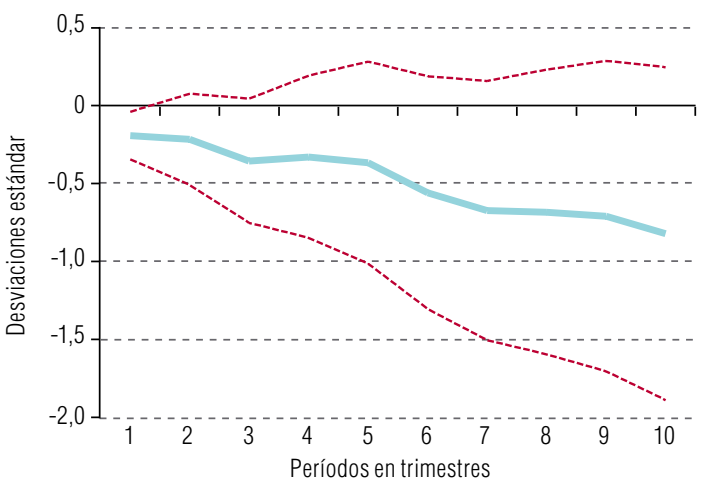

- - - Banda de confianza del 95\%

E. Respuesta del factor agregados monetarios ante el índice de precios de importaciones

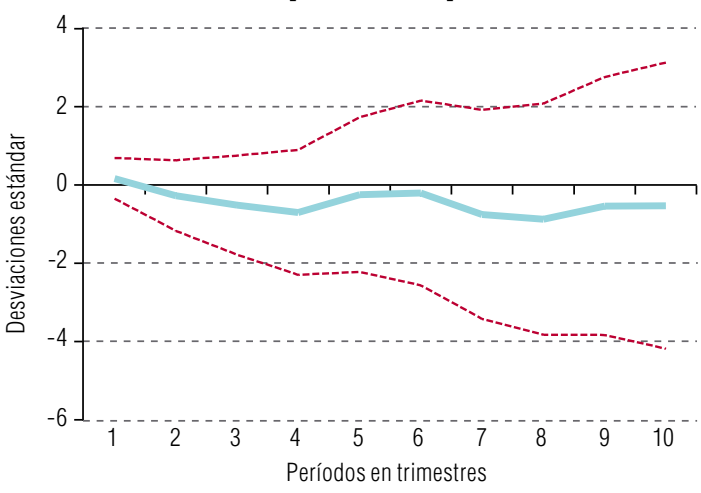

- - - Banda de confianza del 95\% $=$ Agregados monetarios
D. Respuesta del facor tipo de cambio ante el índice de precios de importaciones

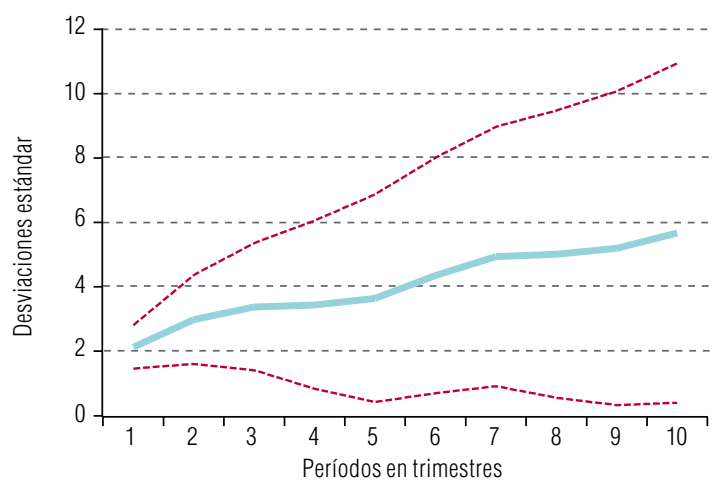

- - - Banda de confianza del 95\% —Tipo de cambio

F. Respuesta del factor precios ante el índice de precios de importaciones

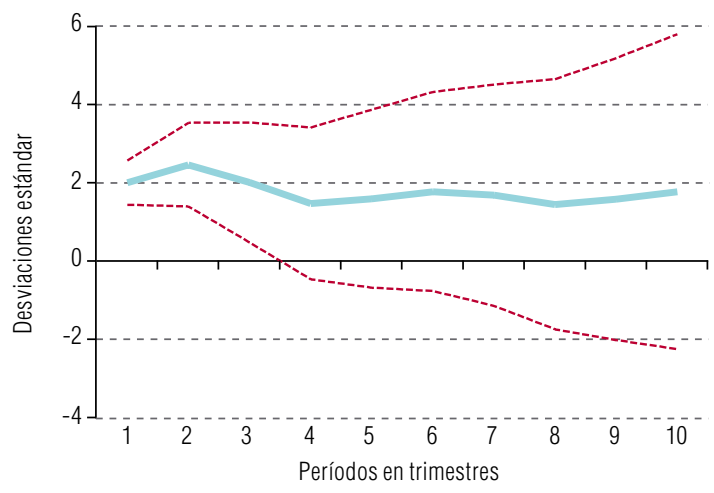

- - - Banda de confianza del 95\% — Precios

G. Respuesta del consumo total ante el índice de precios de importaciones

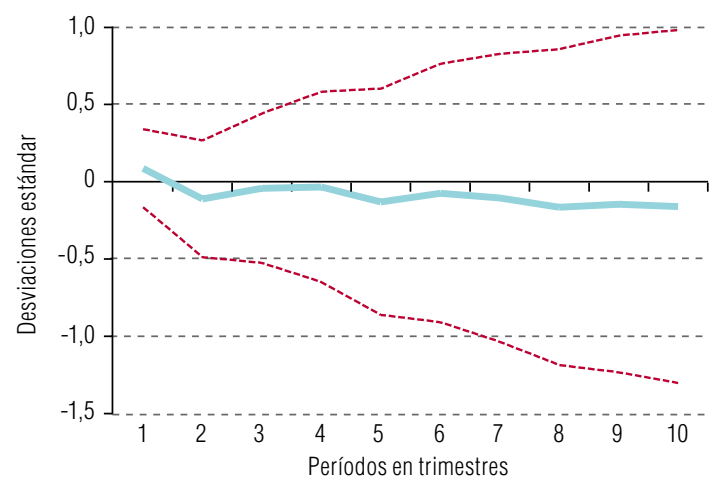

- - - Banda de confianza del 95\% Consumo total

Fuente: Elaboración propia. 
Cuadro A3.1

Descomposición de la varianza de las variables de interés seleccionadas

\begin{tabular}{lcc}
\hline Variables & Porcentaje de la varianza explicada por IPE & Porcentaje de la varianza explicada por IPI \\
\hline Actividad económica (PIB) & 4,00 & 2,00 \\
\hline Consumo total & 3,00 & 3,00 \\
\hline Agregados monetarios & 2,00 & 3,00 \\
\hline Balanza comercial & 4,00 & 1,00 \\
\hline Precios (inflación) & 1,00 & 7,00 \\
\hline Tipo de cambio real & 1,00 & 2,00 \\
\hline Inversión & 6,00 & 5,00 \\
\hline
\end{tabular}

Fuente: Elaboración propia.

Nota: IPE es el índice de precios de exportaciones; IPI es el índice de precios de importaciones. 